\title{
The cardioprotective effect of dexrazoxane (Cardioxane) is consistent with sequestration of poly(ADP-ribose) by self-assembly and not depletion of topoisomerase $2 B$
}

\author{
Keith McCormack \\ McCormack Pharma, a division of McCormack Ltd, Stirling House, 9 Burroughs Gardens, London NW4 4AU, UK \\ Correspondence to: Keith McCormack. Email: KeithMcCormack@mccormack-group.com
}

\begin{abstract}
Following systematic scrutiny of the evidence in support of the hypothesis that the cardioprotective mechanism of action of dexrazoxane is mediated by a 'depletion' or 'downregulation' of Top2 $\beta$ protein levels in heart tissue, the author concludes that this hypothesis is untenable. In seeking to understand how dexrazoxane protects the heart, the outcomes of a customised association rule learning algorithm incorporating the use of antecedent surrogate variables (CEME, 2017 McCormack Pharma) reveal a previously unknown relationship between dexrazoxane and poly(ADP-ribose) (PAR) polymer. The author shows how this previously unknown relationship explains both acute and long-term cardioprotection in patients receiving anthracyclines. In addition, as a direct inhibitor of PAR dexrazoxane has access to the epigenome and this offers a new insight into protection by dexrazoxane against doxorubicin-induced late-onset damage [McCormack $\mathrm{K}$, manuscript in preparation]. Notably, through this review article, the author illustrates the practical application of probing natural language text using an association rule learning algorithm for the discovery of new and interesting associations that, otherwise, would remain lost. Historically, the use of CEME enabled the first report of the capacity of a small molecule to catalyse the hybrid self-assembly of a nucleic acid biopolymer via canonical and non-canonical, non-covalent interactions analogous to Watson Crick and Hoogsteen base pairing, respectively.
\end{abstract}

Keywords: dexrazoxane, anthracyclines, cardioprotection, poly(ADP-ribose), topoisomerase 2 $\beta$, epigenome, Watson Crick

Published: $21 / 12 / 2018$

Received: 24/09/2018

ecancer 2018, 12:889 https://doi.org/10.3332/ecancer.2018.889

Copyright: $\odot$ the authors; licensee ecancermedicalscience. This is an Open Access article distributed under the terms of the Creative Commons Attribution License (http://creativecommons.org/licenses/by/3.0), which permits unrestricted use, distribution, and reproduction in any medium, provided the original work is properly cited. 


\section{The acute cardioprotective effect of dexrazoxane}

\section{Introduction}

\section{A brief historical perspective}

Dexrazoxane (ICRF-187) (Figure 1) belongs to the bisdioxopiperazine class of compounds and is a water-soluble ring-closed analogue of the iron chelator ethylenediaminetetraacetic acid (EDTA). Upon hydrolysis, dexrazoxane opens into its EDTA-like form, ADR-925, which is a strong iron chelator with the ability to displace iron bound to an anthracycline [169].

The interest in the bisdioxopiperazines as potential protectors against anthracycline-mediated myocardial damage emerged from a large series of preclinical studies, notably those performed by Herman et al throughout the 1970s and 1980s [170]. Pre-treatment with dexrazoxane reduced the cardiotoxicity and lethality in non-cancer-bearing Syrian golden hamsters receiving daunorubicin [171]. Furthermore, pre-treatment with dexrazoxane was shown to be cardioprotective in doxorubicin- and daunorubicin-treated miniature swine, beagle dogs and rabbits [172-174]. Later, clinical studies in humans confirmed the cardioprotection exerted by dexrazoxane.

Historically, many mechanisms have been proposed to explain the molecular basis of anthracycline-induced cardiotoxicity. However, the most-investigated mechanism describes the ability of this class of drugs to disrupt iron metabolism and generate an excess of reactive oxygen species (ROS) within cardiomyocytes, with DNA damage resulting from increased oxidative stress and lipid peroxidation [175-178]. From a molecular perspective, the chemical structure of doxorubicin is particularly prone to the generation of free radicals as doxorubicin can be reversibly reduced to a semiquinone, an unstable metabolite whose futile cycling within mitochondria releases ROS [179]. More generally, as a class-effect anthracycline-dependent oxidative stress has long been ascribed to the ability of these drugs to chelate free iron, thereby forming iron-anthracycline complexes which, in turn, react with oxygen and trigger ROS production [180, 181]. Overall, numerous studies emphasise the critical role of free iron in anthracycline-induced cardiotoxicity and suggest that reduction of iron levels may constitute an effective strategy to prevent anthracycline-induced heart failure.

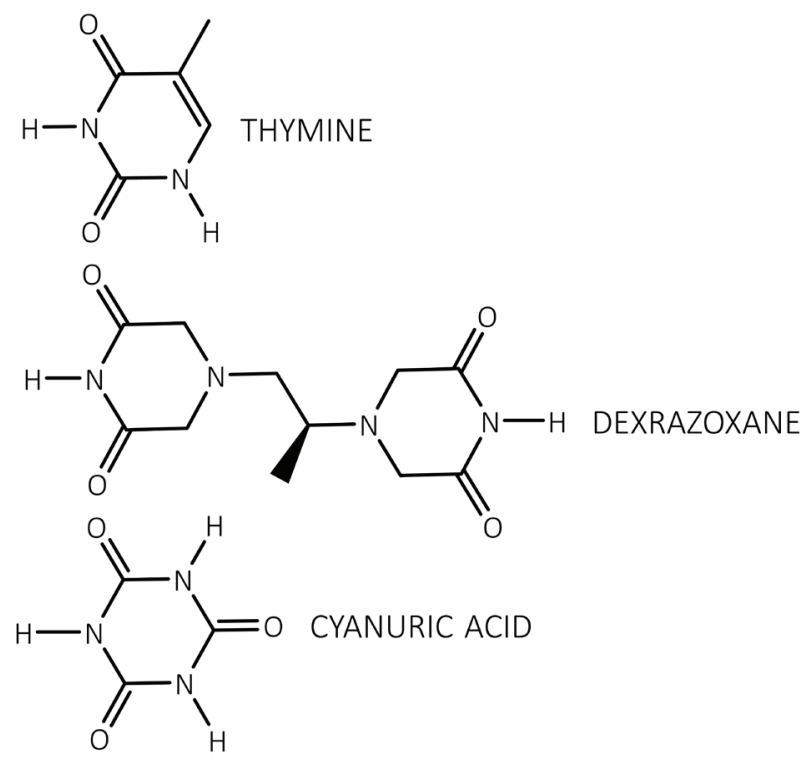

Figure 1. Each dioxopiperazine moiety of dexrazoxane contains a thymine face and cyanuric acid has three thymine faces. 
However, numerous antioxidants and prototypic ROS scavengers that include $\mathrm{N}$-acetylcysteine, coenzyme Q, vitamins $\mathrm{C}$ and $\mathrm{E}$, and several iron chelators have been tested in vitro and in vivo with variable outcomes [64]. None of these compounds have matched or even surpassed the effectiveness of dexrazoxane in chronic anthracycline cardiotoxicity settings, despite being stronger chelators or antioxidants.

By way of illustration, deferiprone is an orally effective $\alpha$-ketohydroxypyridine iron chelator. As a small lipophilic molecule, deferiprone penetrates readily into various tissues and has been shown to remove excess iron from the heart [182] and bind labile free and accumulated cellular iron within mitochondria and lysosomes [183]. Nevertheless, using a clinically relevant and dexrazoxane-validated model of chronic anthracycline-induced cardiotoxicity in rabbits [184], deferiprone showed no potential to alleviate either anthracycline-induced oxidative stress or left ventricular cardiac damage and congestive heart failure, as assessed by both echocardiography and left ventricular catheterisation [185]. Deferasirox, a clinically approved iron chelator likewise failed to protect isolated rat cardiomyocytes against doxorubicininduced damage despite the observations that this drug rapidly entered cardiomyocytes and efficiently removed iron from its complex with doxorubicin [186].

In summary, studies with various iron-specific chelators (both clinically approved drugs as well as experimental agents) have yielded rather mixed results, particularly when evaluated using clinically relevant animal models of anthracycline-induced cardiotoxicity. Notably, none of these agents achieved the model-independent protective efficiency of dexrazoxane even though the studied agents are even stronger and more selective intracellular iron chelators than dexrazoxane.

Vavrova et al [64] investigated the involvement of Top2 in anthracycline-induced cardiotoxicity. Using primary cultures of isolated rat neonatal ventricular cardiomyocytes, they examined the protective effects of dexrazoxane against cardiotoxicity induced by daunorubicin and doxorubicin. From the outcomes of this meticulous and systematic investigation, these workers conclude that, in agreement with previous suggestions, the cardioprotective effect of dexrazoxane is not adequately explained by iron chelation or antioxidant properties. Rather, in consideration of reports of the role of Top2 $\beta$ in mediating anthracycline-mediated cardiotoxicity [1-3], notably that by Zhang et al [1] and Lyu et al [4], they propose that the selective degradation of Top2 $\beta$ is a plausible explanation for the cardioprotective effects of dexrazoxane.

\section{Top2 $\beta$ is the molecular target of doxorubicin}

Anthracyclines, notably doxorubicin are widely used chemotherapeutic agents. However, and as briefly discussed above, their clinical use is often limited by dose-dependent cardiotoxicity. Zhang et al hypothesised that doxorubicin-induced cardiotoxicity is mediated by Top2 $\beta$, the only Top2 enzyme expressed in cardiomyocytes [1]. These workers elegantly show that cardiomyocyte-specific deletion of Top2 $\beta$ (encoding Top2 $\beta$ ) protects cardiomyocytes from doxorubicin-induced DNA double-strand breaks (DSBs) and transcriptome changes that are responsible for defective mitochondrial biogenesis and the generation of ROS. Importantly, they also show that cardiomyocyte-specific deletion of Top $2 \beta$ protects mice from the development of doxorubicin-induced progressive heart failure, providing robust support for the view that doxorubicin-induced cardiotoxicity is mediated by Top $2 \beta$ in cardiomyocytes. They conclude that the molecular phenotype of acute and chronic doxorubicin cardiomyopathy is characterised by the formation of a ternary DNA-Top2 $\beta$-doxorubicin cleavage complex, that triggers DSBs in DNA, in part by preventing religation of Top2 $\beta$-induced strand breaks.

In accord with these erudite observations, Velpongsa and Yeh [2] propose that inhibiting and deleting Top2 $\beta$ in the heart should be tested as a strategy for the primary prevention of anthracycline-induced cardiotoxicity, and in a recent review, Moudgil and Yeh [3] conclude that Top $2 \beta$ is required to initiate the entire phenotypic cascade of doxorubicin-induced cardiomyopathy.

\section{Dexrazoxane induces degradation of Top $2 \beta$}

Several investigators have observed depletion of the doxorubicin target Top $2 \beta$ in cells treated with dexrazoxane. From their results using H9C2 cardiomyocytes, Lyu et al [4] suggest that dexrazoxane may prevent doxorubicin-induced DNA damage by triggering proteasomal degradation of Top $2 \beta$. They argue that dexrazoxane binds to theTop $2 \beta$-DNA binary complex and stabilises Top $2 \beta$ as a closed clamp upon DNA. This clamp blocks the movement of the transcription-elongation complex resulting in transcriptional arrest, which, in turn, triggers $26 \mathrm{~S}$ proteasome-dependent degradation of Top2 $\beta$, a well-documented mechanism for clearing Top2-mediated 'transcriptionroad blocks' [6-8]. 
Deng et al [42] report depletion of Top2 $\beta$ protein in the hearts of C57BL (B6) mice following the intraperitoneal administration of dexrazoxane. Together with the results of additional systematic and complementary in vitro investigations, these workers describe a model in which dexrazoxane depletes cardiac Top $2 \beta$, thereby reducing the substrate for 'doxorubicin poisoning'. Accordingly, subsequent administration of doxorubicin is anticipated to effect lower levels of DNA damage by comparison with no dexrazoxane pre-treatment.

A multidisciplinary collaborative effort between the Faculties of Pharmacology, Pharmacy and Medicine at Charles University in Hradec Králové, Czech Republic [162-164], was undertaken to investigate the molecular basis for the cardioprotective effect of dexrazoxane using rabbits together with $\mathrm{H} 9 \mathrm{C} 2$ cells and freshly harvested ventricular rat cardiomyocytes. From the results of this conjoint study, the workers report that in rabbits, pre-treatment with dexrazoxane completely prevents daunorubicin-induced heart damage. Notably, this protection was unrelated to an effect upon oxidation status and dexrazoxane was shown to exert no effect upon mobilisation of intracellular iron. However, experiments both in vivo and in vitro showed a decrease in Top2 $\beta$ protein levels within rabbit heart and cell cultures following exposure to dexrazoxane. Taken together, they conclude that their findings support the view that the cardioprotective effects of dexrazoxane may not be attributable to iron chelation and/or mitigation of oxidative stress; rather, they suggest that depletion of Top2 $\beta$ may represent the molecular basis for the cardioprotective action of dexrazoxane.

In a series of National Institute of Health funding applications for the fiscal years 2015, 2016, 2017 and 2018, Yeh provides details of his proposed research aims that include a determination of whether dexrazoxane-induced degradation of Top2 $\beta$ in the heart prevents doxorubicininduced cardiotoxicity, and by what mechanism does dexrazoxane induce degradation of Top2 $\beta$ [9, 165-168]. Within the formal abstracts of these applications, Yeh alludes to preliminary studies in which he and his coworkers showed that dexrazoxane induced degradation of Top2 $\beta$ through a proteasome-dependent mechanism. Indeed, the outcomes of studies detailed within the earlier Patent Application US $14 / 155,858$ are presented by the same worker in support of the claim therein that the cardioprotective compound dexrazoxane functions in some part by reducing Top $2 \beta$ protein levels in heart tissue [5]. Interestingly, within that application, the inventor showed that dexrazoxanemediated Top2 $\beta$ degradation was inhibited by the proteasome inhibitor MG132 suggesting a proteasome-dependent mechanism for the effects of dexrazoxane upon the level of Top $2 \beta$ protein expression.

Collectively, the outcomes presented above are seductive and in part have given rise to the burgeoning hypothesis, evident within an abundant scientific and clinical literature that dexrazoxane protects the heart against anthracycline-induced damage by promoting the proteasomal degradation of Top $2 \beta$, the Top 2 isoenzyme that predominates in cardiomyocytes.

\section{Does the cardioprotective mechanism of dexrazoxane involve Top2 $\beta$ ?}

In summary, the evidence in support of a pivotal role of Top2 $\beta$ in mediating doxorubicin-induced cardiotoxicity and heart failure is robust and convincing.

However, as this systematic analysis shows the presumed corollary that the cardioprotective mechanism of action of dexrazoxane is mediated by a 'depletion' or 'downregulation' of Top2 $\beta$ protein levels in heart tissue is untenable and incorrect. Indeed, classically, this 'new hypothesis' is a category error that contains the fallacy of division (assumes that the part has the properties of the whole).

If dexrazoxane protects the heart against doxorubicin-induced damage by suppressing Top $2 \beta$ protein levels in heart tissue, then two criteria must be satisfied absolutely and unequivocally:

- Following a single infusion of dexrazoxane, Top2 $\beta$ protein levels in heart tissue must be suppressed to, or below some threshold whereby Top $2 \beta$ protein levels no longer represent a molecular target for the effects of doxorubicin.

- Dexrazoxane-suppressed Top2 $\beta$ protein levels in heart tissue must remain at, or below the above threshold during the period that the heart is exposed to damaging levels of doxorubicin.

In seeking to establish whether these criteria are satisfied, scrutiny of the available literature is necessary to establish the period during which the levels of doxorubicin within heart tissue can be reasonably expected to be toxic following single-dose administration. Within this window of cardiotoxicity, it remains to determine whether a single dose of dexrazoxane administered prior to doxorubicin can suppress levels of Top2 $\beta$ protein levels in heart tissue to or below a threshold at which they do not represent a molecular target for doxorubicin for the entire period during which doxorubicin residence poses a threat to cardiomyocyte viability. 


\section{Accumulation of doxorubicin within the heart}

\section{Study duration 24 hours}

Using Friend Leukaemia Virus B (FVB) mice, van Asperen's group report mean doxorubicin concentrations of 21.5 , 12.6 and 1.7 $\mu \mathrm{mol} \mathrm{kg} \mathrm{kg}^{-1}$ in the hearts of mice sacrificed at 1,4 and 24 hours, respectively, following the administration of doxorubicin into the tail vein at a dose of $5 \mathrm{mg} \mathrm{kg}^{-1}$ [10]. Nwankwoala et al studied the pharmacokinetics and tissue distribution of doxorubicin in Wistar rats.

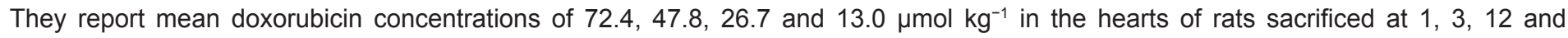
24 hours, respectively, following the intravenous administration of doxorubicin at a dose of $20 \mathrm{mg} \mathrm{kg}^{-1}$. Administering doxorubicin at a dose of $1 \mathrm{mg} \mathrm{kg}^{-1}$ they report mean doxorubicin concentrations of $6.7,2.8$ and $1.5 \mu \mathrm{mol} \mathrm{kg}^{-1}$ in the hearts of rats sacrificed at 1,12 and 24 hours, respectively [11].

Using Sprague-Dawley rats, Rahman et al [12] compared the pharmacokinetic and tissue disposition of doxorubicin with that of doxorubicin encapsulated within cardiolipin-modified liposomes. They report mean doxorubicin concentrations of $27.8,21.2,18.03$ and $8.6 \mu \mathrm{mol} \mathrm{kg}^{-1}$ in the hearts of rats sacrificed at 30 minutes, 2, 4 and 24 hours, respectively, following the administration of free doxorubicin into the femoral vein at a dose of $6 \mathrm{mg} \mathrm{kg}^{-1}$. At the same time, they report mean cardiac concentrations of $16.2,16.0,10.7$ and $4.2 \mu \mathrm{mol} \mathrm{kg}{ }^{-1}$, respectively, following the administration of the liposomal-formulated doxorubicin at a dose of $6 \mathrm{mg}$ (doxorubicin) $\mathrm{kg}^{-1}[12]$.

\section{Study duration 48 hours}

Ozols et al [13] studied the tissue disposition of doxorubicin in C3HeB/FeJ mice with a transplantable ovarian cancer. They report mean doxorubicin concentrations of $18.4,7.4$ and $1.2 \mu \mathrm{mol} \mathrm{kg}{ }^{-1}$ in the hearts of mice that were sacrificed at 15 minutes, 24 and 48 hours, respectively, following the administration of doxorubicin at an LD10 (lethal dose to $10 \%$ of animals) dose of $10 \mathrm{mg} \mathrm{kg}^{-1}$ into the tail vein (numerical data extracted by digitisation of graphical plots).

More recently, Staples et al [14] studied the distribution of doxorubicin following the use of low-frequency ultrasound to trigger the release of micelle-encapsulated doxorubicin in Berlin Druckrey strain IX (BDIX) rats with bilateral leg tumours following injection of the DHD/K12/ $\mathrm{TRb}$ colorectal cell line. They report mean doxorubicin concentrations of $10.4,4.7,4.4,3.05,1.6$ and $0.8 \mu \mathrm{mol} \mathrm{kg}^{-1}$ in the hearts of rats that were sacrificed at 30 minutes, 6, 8, 12, 24 and 48 hours, respectively, following the administration of encapsulated-doxorubicin into the lateral tail vein at a dose of $2.67 \mathrm{mg}$ (doxorubicin) $\mathrm{kg}^{-1}$.

\section{Study duration beyond 48 hours}

Staples et al [14] additionally report detectable levels of doxorubicin in the hearts of rats that were sacrificed at 96 and 168 hours. Interestingly, in a subgroup of rats, repeated weekly-administrations of encapsulated doxorubicin over a 4-week period resulted in a significantly greater amount of doxorubicin in the heart by comparison with that following a single treatment $(p=0.044)$, with no statistically significant differences in concentrations between the single and multiple treatment groups in the liver, leg muscle and tumour tissues $(p=0.262, p=$ 0.397 and $p=0.327$, respectively) (numerical data extracted by digitisation of graphical plots). These findings support the view (see below) that the heart selectively accumulates doxorubicin.

Consistent with the report by Staples et al [14] of detectable levels of doxorubicin at long time points, Chenard [15] reported detectable levels of doxorubicin at 192 hours in the hearts of Sprague Dawley rats following the intraperitoneal administration of doxorubicin at a dose of either 1.5 or $4.5 \mathrm{mg} \mathrm{kg}^{-1}$.

\section{Long-term retention of doxorubicin within the heart (animal data)}

The persistence of doxorubicin within myocardial cells had been previously demonstrated by the early histofluorescence study by Amin et al [16]. Following the intravenous administration of doxorubicin at a dose of $15 \mathrm{mg} \mathrm{kg}^{-1}$ to Sprague Dawley rats, fluorescence emission was observed in myocardial cells of the left ventricle until 28 days after administration. 


\section{Long-term retention of doxorubicin within the heart (patient data)}

In accord with the outcomes from animal studies, Stewart et al [17] demonstrated the prolonged retention of doxorubicin within autopsy cardiac tissue from 35 patients who had received doxorubicin at any time antemortem. The median lifetime cumulative doxorubicin dose was $268 \mathrm{mg} \mathrm{m}^{-2}$ (range 30-670 $\mathrm{mg} \mathrm{m}^{-2}$ ), and the median time from the last doxorubicin treatment to death was 81 days (range, 1-931 days). The median doxorubicin concentration in cardiac tissue was $0.11 \mu \mathrm{mol} \mathrm{kg}{ }^{-1}$ (range, 0-3.06 $\mu \mathrm{mol} \mathrm{kg}^{-1}$ ). These workers add that their results suggest that cardiomyocytes selectively accumulate doxorubicin by comparison with other types of muscle.

\section{Doxorubicin displays rapid uptake and slow elimination}

Taken together, the data presented above are consistent with a prolonged terminal half-life of doxorubicin and a propensity to accumulate within cardiac tissue. In humans, free doxorubicin has a distributive half-life of about 5 minutes and a terminal half-life of $20-48$ hours, indicating fast uptake into tissues and slow elimination thereafter [18]. High-affinity binding of doxorubicin with the diphosphatidylglycerol lipid, cardiolipin located within the inner mitochondrial membrane, may explain in some part the retention of doxorubicin by the mitochondrionrich cardiomyocytes [19].

\section{Can we determine a cardiotoxic limiting concentration of doxorubicin?}

While the studies reviewed above show measurable concentrations of doxorubicin at extended time points in the hearts of both animals and humans, how can we identify a limiting concentration, below which doxorubicin is not overtly cardiotoxic?

The half-maximal inhibitory concentration (IC50) of doxorubicin as a measure of potency in reducing the viability of cardiomyocytes provides a useful datum for enabling perspective. The author identified ten studies that report IC50 values for growth inhibition of cardiomyocytes using either free or liposomal-encapsulated doxorubicin, with a median value of $1.67 \mu \mathrm{M}: 0.02,0.04,0.12,0.31,1.33,2.0,3.09,3.5$, 3.6 and 5.6 , with units as $\mu \mathrm{M}[20-28]$.

\section{Accumulation of doxorubicin suggests long-term effects on Top2 $\beta$}

Accepting the proportionality error in comparing concentrations in an aqueous buffer $\left(\mathrm{IC} 50, \mu \mathrm{mol} \mathrm{dm}^{-3}=\mu \mathrm{M}\right)$ with concentrations in ex vivo tissue mass $\left(\mu \mathrm{mol} \mathrm{kg}{ }^{-1}\right.$ ), then using the literature values of IC50 as a measure of toxicity, it can be concluded that for prolonged periods the cardiac concentrations of doxorubicin are sufficient to negatively impact upon cardiomyocyte viability. The limited animal data suggest that cardiotoxic levels of doxorubicin remain significantly above the median IC50 value of $1.67 \mu \mathrm{M}$ (see above) for at least 24 hours and decline below this limiting concentration between 24 and 48 hours. In both animals and patients, there is convincing evidence for long-term (days/weeks) retention of doxorubicin within cardiac tissue. Accordingly, given the long-term accumulation of doxorubicin within the heart, if the short pre-administration single infusion of dexrazoxane exerts a cardioprotective effect by depleting Top $2 \beta$ protein, then by necessity suppression of the level of Top2 $\beta$ protein within the heart must be profound and long lasting. As the author argues, this seems a remote prospect given that by comparison with the slow elimination of doxorubicin, dexrazoxane has a remarkably short half-life. The logical corollary of this substantial difference in pharmacokinetics is twofold. First, dexrazoxane must exert long-lasting suppression that results in maintenance of Top $2 \beta$ protein below threshold. Second, in the absence of long-lasting suppression, recovery of suppressed Top $2 \beta$ protein (transcription) must be slow.

\section{Does dexrazoxane eliminate cardiac Top2 $\beta$ as a target?}

If dexrazoxane protects the heart against doxorubicin-induced damage by suppressing Top $2 \beta$ protein levels, then Top $2 \beta$ protein levels in heart tissue must be suppressed to, or below some threshold whereby Top $2 \beta$ protein levels no longer represent a molecular target for the effects of doxorubicin. As a prelude to reviewing the literature reports that describe the suppression of cardiac Top $2 \beta$ protein levels by 
dexrazoxane, detailed attention must be given to the pharmacokinetics of both dexrazoxane and doxorubicin since the duration of any suppressive effects will be determined by the residence time of dexrazoxane and the natural turnover of Top2 $\beta$ protein.

\section{Dexrazoxane is slowly metabolised in vitro}

Under physiological conditions $\left(37^{\circ} \mathrm{C}\right.$ and $\left.\mathrm{pH} 7.4\right)$ in vitro, dexrazoxane is slowly hydrolysed to the single-ring-opened metabolites (described elsewhere as B and C) ( $t_{1 / 2}$ of 9.3 hours), and to the final hydrolysis product ADR-925 ( $t_{1 / 2}$ of 23 hours) [29-32]. Given the slow rate at which dexrazoxane hydrolysis occurs in vitro, considerable caution must be exercised in extrapolating laboratory findings using cell preparations exposed continuously to high concentrations of dexrazoxane, to the clinical setting with rapidly declining concentrations of dexrazoxane.

\section{In a clinical setting, the metabolism of dexrazoxane is extremely rapid}

Schroeder et al [33] investigated the metabolism of dexrazoxane in cancer patients with brain metastases treated with high-dose etoposide. They showed that the two single-ring-opened hydrolysis intermediates of dexrazoxane, B and C appeared in the plasma at low levels upon completion of the dexrazoxane infusion and then rapidly decreased with half-lives of 0.6 and 2.5 hours, respectively. A plasma concentration of $10 \mu \mathrm{M}$ of ADR-925 was also detected at the completion of the dexrazoxane intravenous infusion period, indicating, as these workers conclude that in this setting, dexrazoxane is rapidly metabolised.

\section{In humans, the terminal half-life of doxorubicin is up to 48 hours and that for dexrazoxane is}

\section{approximately 2 hours}

In humans, free doxorubicin has a distributive half-life of about 5 minutes and a terminal half-life of 20-48 hours, indicating fast uptake into tissues and slow elimination thereafter [18]. By contrast, after intravenous administration to patients with cancer plasma concentrations of dexrazoxane were observed to decline with an average half-life of $2.2+/-0.42$ hours [34].

In the phase I study by Earhart et al [35], following the administration of dexrazoxane as a 30-minute infusion, at the final time point of 15 hours (approximately seven dexrazoxane half-lives) concentration-time curves show that the mean plasma concentration of dexrazoxane is at the limit of detection. Given that dexrazoxane is $<2 \%$ bound to plasma proteins [34], and that the uncharged parent compound rapidly equilibrates across the cell membranes [36], then intracellular concentrations will likewise be at or near zero. Moreover, since it is only the parent compound that is a catalytic inhibitor of Top2 $\beta$ [37-40], then undegraded Top2 $\beta$ protein remaining at 15 hours, together with newly synthesised Top $2 \beta$ protein, will represent an exclusive target for doxorubicin, which at 15 hours is present at a very substantial concentration within cardiac tissues [10-19].

\section{Partial suppression of Top $2 \beta$ protein at early times in cardiomyocytes}

Lyu et al [4] treated H9C2 rat cardiomyocytes with 100- $\mu \mathrm{M}$ dexrazoxane for 0, 1, 2, 4 and 6 hours. Following incubation, cells were lysed and protein levels of Top $2 \alpha$ and Top $2 \beta$ were determined by western blotting followed by detection using enhanced chemiluminescence using X-ray films or the Kodak Image Station 2000R. These visualisations show a time-dependent disappearance of Top2 $\beta$, but with no observable effect upon the levels of Top2 $\alpha$. Yan et al [41] treated HTETOP cells with 100- $\mu \mathrm{M}$ dexrazoxane for 0, 3, 6, 12 and 24 hours; and in a parallel assay, cells were incubated for 24 hours with 1-, 5-, 25-, 100-, and 200- $\mu \mathrm{M}$ dexrazoxane. Following incubation, cells were lysed and protein levels of Top $2 \alpha$ and Top2 $\beta$ were determined by western blotting with detection using enhanced chemiluminescence followed by densitometric analysis. The western blot images show that at all time points incubation with dexrazoxane resulted in partial suppression of Top $2 \beta$ protein levels. Paradoxically, at 24 hours, cells incubated with $200-\mu \mathrm{M}$ dexrazoxane showed markedly less depletion of Top $2 \beta$ protein by comparison with cells exposed to concentrations of dexrazoxane within the range 5-100 $\mu \mathrm{M}$ for the same period. In summary, and repeating the concern expressed earlier, considerable caution must be exercised in extrapolating laboratory findings using cell preparations exposed continuously to high concentrations of dexrazoxane, to the clinical setting with rapidly declining concentrations of dexrazoxane. 


\section{Suppression of Top2 $\beta$ protein levels is partial and transient}

The western blot images presented by Deng et al [42] reveal mean Top2 $\beta$ protein expression of $47 \%$, 32\% and $64 \%$ in the hearts of C57BL (B6) mice that were sacrificed at 6, 24 and 48 hours, respectively, following the intraperitoneal administration of $200 \mathrm{mg} \mathrm{kg}^{-1} \mathrm{dexrazoxane}$ with the control group of mice receiving saline injections. According to Deng et al, the increased expression of Top2 $\beta$ protein observed at 48 hours indicates recovery from the depletion induced by dexrazoxane. In parallel experiments, mice were injected with 200 mg $\mathrm{kg}^{-1}$ dexrazoxane alone, $20 \mathrm{mg} \mathrm{kg}^{-1}$ doxorubicin alone or in combination whereby dexrazoxane was injected 30 minutes before doxorubicin; hearts were removed 24 hours later for western blot analysis. Relative to the control group, the mean Top $2 \beta$ protein expression was $63 \%$, $95 \%$ and $73 \%$ in mice that received dexrazoxane, doxorubicin and a combination of both drugs, respectively. In reviewing their results, Deng et al conclude that the depletion of Top $2 \beta$ protein by dexrazoxane in vivo is 'transient'. They speculate that transient depletion of Top $2 \beta$ protein by dexrazoxane reduces the risk or the duration of any adverse effects upon cardiomyocyte viability associated with loss of the Top2 $\beta$ enzyme.

\section{Rapid recovery of suppressed Top2 $\beta$ protein levels}

The effect of dexrazoxane on Top2 $\beta$ protein levels was studied by Yeh [5] in mice following the intraperitoneal administration of 100 mg $\mathrm{kg}^{-1}$ dexrazoxane. Western blotting of harvested hearts shows that Top2 $\beta$ protein was 'nearly undetectable' [5] at 6 hours after the administration of dexrazoxane, but recovery of Top $2 \beta$ protein is visibly evident at the next time point of 12 hours.

\section{Degradation of Top $2 \beta$ protein levels is not unique to dexrazoxane}

Preferential degradation of Top2 $\beta$ is a ubiquitous phenomenon that is observed using both catalytic inhibitors and Top2 poisons and is, by no means, unique to dexrazoxane. This much-observed effect has been reported in studies using VM-26 (teniposide), VM-16 (etoposide), ICRF-193, BNS-22, genistein, lycobetaine, bufalin and dexrazoxane, and in an array of cell types [4, 6, 7, 41-56, 116]. Despite the plethora of drugs that degrade Top $2 \beta$ protein, dexrazoxane remains the only product that is licenced for the prevention of cardiotoxicity associated with the use of anthracyclines; a posteriori, in isolation, this clinical fact provides presumptive evidence against any involvement of the Top2 $\beta$ protein in the cardioprotective mechanism of dexrazoxane.

\section{Top2 $\beta$ protein remains a target even at near-zero levels}

Kersting et al [57] investigated if the expression of Top $2 \alpha$ and Top2 $\beta$ enzymes in normal cells display variability between individuals and whether this variability affects the apoptotic response to the effects of doxorubicin. They tested their hypothesis using peripheral blood leucocytes, a class of cells that has been shown to undergo apoptosis in response to doxorubicin [58, 59]. Peripheral blood leucocytes were isolated from healthy volunteers and exposed to 1- $\mu \mathrm{M}$ doxorubicin ex vivo for 24 hours. Apoptotic cells were detected by staining with a fluorescent conjugate of Annexin $\mathrm{V}$, a protein that has a high affinity for binding to the early apoptosis marker, translocated membrane phosphatidylserine. Total Top2 expression was measured as catalytic activity using an assay that measures the Top2specific enzymatic conversion of highly catenated kDNA into nicked circular and relaxed circular DNA. Resolution of Top2 isoforms was undertaken using protein extracted from the nuclei of peripheral leucocytes followed by western blot analysis using Top $2 \alpha$ and Top $2 \beta$ antibodies.

Of the two human Top2 proteins, only Top2 $\beta$ was detected by western blotting of the nuclear extracts of peripheral blood leucocytes. Strikingly, Top2 $\beta$ catalytic activity correlated significantly $(p=0.01)$ with the apoptotic response in peripheral blood leucocytes exposed to $1-\mu \mathrm{M}$ doxorubicin (Figure 2). As discussed below, this correlation has a profound significance in refuting the argument that dexrazoxane exerts a cardioprotective effect by lowering Top $2 \beta$ protein levels within heart tissue.

However, before assessing the potential implications of this finding by Kersting et al [57], it is useful and necessary to explore the goodnessof-fit of these data. The increased variance above the median value of the abscissa (top right-hand corner) suggests that the correlation 
can be improved by weighting and exploring a nonlinear regression. Using the subscriber version of MyCurveFit, following extraction of the original data by digitisation, a weighted power curve regression yields $R=0.786$ with $p=0.0003$. Residual plot analysis (not shown here) confirms that the data of Kersting et al [57] can be represented adequately by both a linear regression and a weighted nonlinear regression. Notably, both regressions provide the same take-home message which is that at levels of Top2 $\beta$ protein expression close to zero $(<0.05$ as enzyme units) (Figure 2), there is clear evidence of substantial apoptosis $(30 \%)$ following exposure to doxorubicin. Accepting these data, it is not unreasonable to conclude that if the cardioprotective effect of dexrazoxane is mediated by a dexrazoxane-induced lowering of Top $2 \beta$ protein levels then levels of Top $2 \beta$ protein need to be suppressed to near-zero levels and remain that way for a prolonged period (days and weeks), and with no provision for early recovery. However, as the animal and human data reviewed above indicate, that does not happen.

\section{Is the use of peripheral blood leucocytes relevant to the effects of doxorubicin within cardiac tissue?}

Ten years after the seminal contribution by Kersting et al [57], Lipshultz et al [60] studied the mitochondrial DNA function in doxorubicintreated childhood acute lymphoblastic leukaemia (ALL) survivors. From the outcomes of this long-term follow-up study, they cautiously propose that functional similarities may exist between the mitochondria of peripheral blood mononuclear cells (PBMCs) and those of cardiomyocytes and that the transcriptome of PBMCs might serve as a surrogate marker of doxorubicin-induced cardiotoxicity. Moreover, preliminary results by Yeh [5] are used in support of his proposal that Top2 $\beta$ protein levels of peripheral blood can be used to predict a patient's susceptibility to anthracycline-induced cardiotoxicity [5].

Taken together, the author concludes that the earlier work by Kersting et al [57] is relevant to this analysis and the outcomes of that work permit the extrapolation that in the heart, near-zero levels of Top2 $\beta$ protein are a sufficient and functional target for doxorubicin.

In summary, there are no published data to show that in clinical practise dexrazoxane attenuates or compromises the cardiotoxicity of doxorubicin through any significant effects upon the levels of doxorubicin's target, Top2 $\beta$ protein, and as presented next, new findings show that dexrazoxane is cardioprotective even when dexrazoxane-induced decreases in Top2 $\beta$ protein are blocked.

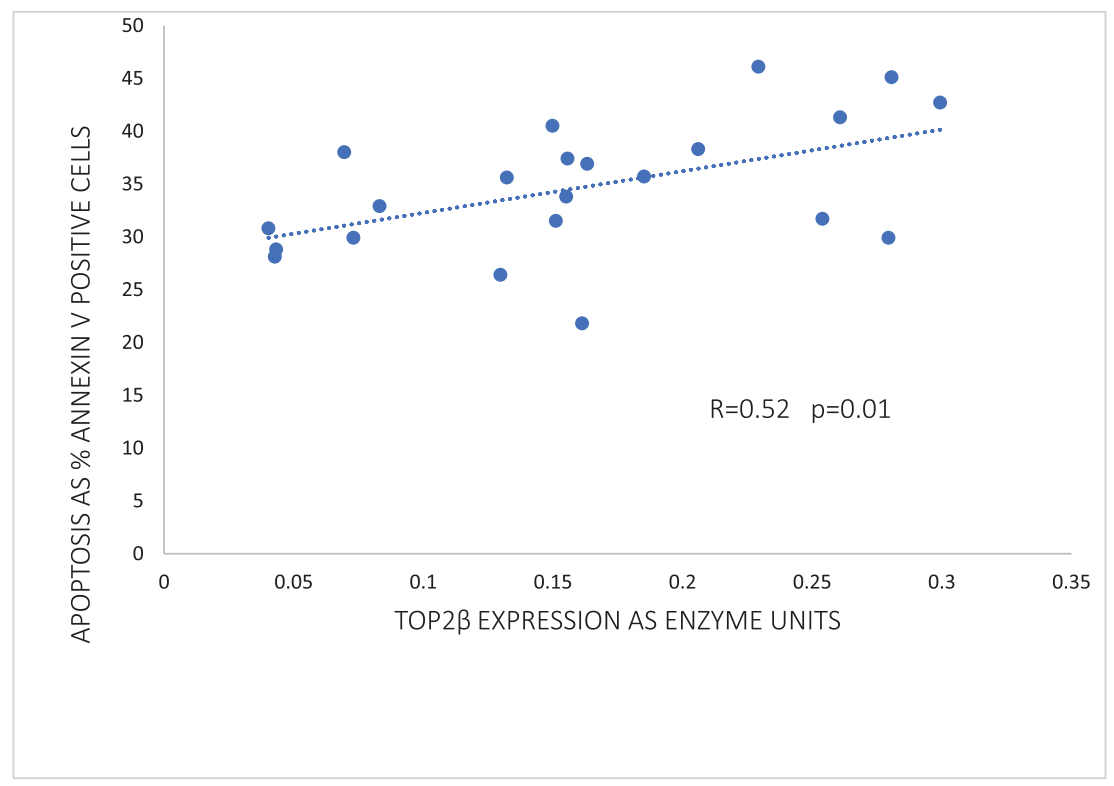

Figure 2. Correlation between Top2 $\beta$ expression and the percentage of apoptotic cells in peripheral blood leucocytes of 22 healthy volunteers following 24 hours ex vivo incubation with 1- $\mu \mathrm{M}$ doxorubicin. Data adapted from Kersting et al [57]. 


\section{New findings: Dexrazoxane is cardioprotective even when degradation of Top2 $\beta$ is blocked}

Using their well-validated neonatal ventricular myocyte model, Hasinoff et al [61] investigated the cardiotoxicity of the proteasome inhibitors, bortezomib and carfilzomib alone and in combination with doxorubicin. While they show that bortezomib and carfilzomib were toxic to myocytes and induced apoptosis, they also observed that a brief pre-exposure of myocytes to nontoxic concentrations of bortezomib or carfilzomib markedly increased doxorubicin-mediated damage. Accordingly, they propose that in their model, the combination of doxorubicin with either bortezomib or carfilzomib may produce more than additive cardiotoxicity. Moreover, they add that these new results are worrying since the clinical use of doxorubicin, combined with either bortezomib or carfilzomib could potentially induce greater cardiotoxicity than the individual drugs used alone.

Importantly, the methodology of this study offers a unique opportunity to additionally investigate the mechanism of action of dexrazoxane.

With the use of proteasome inhibitors, Hasinoff et al [61] tested the 'Top2 $\beta$ hypothesis' by determining whether inhibition of dexrazoxaneinduced proteasomal degradation of Top $2 \beta$ could block the ability of dexrazoxane to protect against doxorubicin-induced myocyte damage. That is, if dexrazoxane exerts its protective effects against doxorubicin-induced myocyte damage by inducing proteasomal Top $2 \beta$ degradation, then inhibition of the dexrazoxane-induced proteasomal degradation of Top2 $\beta$ through prior treatment with bortezomib or carfilzomib would be expected to eliminate or reduce the protective effects of dexrazoxane.

The effect of pre-treating myocytes with the proteasomal inhibitors, bortezomib or carfilzomib upon the dexrazoxane-induced reduction in Top2 $\beta$ protein levels are shown in Figure 3. Both inhibitors effectively prevented the dexrazoxane-induced proteasomal degradation of Top2 $\beta$.

Having established that the proteasomal inhibitors effectively prevented the dexrazoxane-induced proteasomal degradation of Top2 $\beta$, Hasinoff et al treated myocytes with a proteasomal inhibitor, a proteasomal inhibitor plus dexrazoxane, a proteasomal inhibitor plus doxorubicin or a proteasomal inhibitor plus doxorubicin and dexrazoxane. The damage was measured using the lactate dehydrogenase release assay, which is a widely used measure of drug-induced damage to cardiomyocytes [62,63], and which has been validated as a sensitive and reliable assay for measuring anthracycline-induced injury and the protective effects of dexrazoxane upon neonatal rat ventricular cardiomyocytes [64].

As the outcomes from this elegant study show, despite the effective blockade of the dexrazoxane-induced proteasomal degradation of Top $2 \beta$, the protective effects of dexrazoxane were largely maintained against the damaging effects of doxorubicin over a series of bortezomib and carfilzomib concentrations. Hasinoff et al conclude that 'These results suggest that dexrazoxane does not protect against doxorubicin-mediated damage solely by reducing topoisomerase II $\beta$ levels in the heart...'

Moreover, they add that it is noteworthy that other workers using a rat model have also demonstrated that dexrazoxane prevents carfilzomib-induced cardiotoxicity, providing further support for a mechanism of action that is unrelated to a dexrazoxane-induced proteasomal degradation of Top2 $\beta[65,66]$.

\section{Summary}

Following a near-exhaustive survey of the literature, the evidence fails to support the assertion that the clinical cardioprotective effect of dexrazoxane is mediated by a dexrazoxane-induced lowering of the level of Top2 $\beta$ protein. At best, the in vivo results show that the lowering of Top $2 \beta$ protein level by dexrazoxane is transient and short-lived (hours) with a rapid recovery by newly synthesised Top $2 \beta$ protein. Within the context of persistently elevated levels of doxorubicin over a period of days and weeks, it is difficult to understand how in a clinical setting lowering of Top $2 \beta$ protein levels following a single 15-minute infusion of dexrazoxane, with a mean half-life of 2.2 hours [34], can translate into a cardioprotective effect.

Moreover, such a reductionist notion of Top2 $\beta$ protein lowering also fails to explain the long-term (years) cardioprotective effects of dexrazoxane [67] or the quantifiable differences in cell biology between long-term survivors who received doxorubicin by comparison with those who received doxorubicin plus dexrazoxane [60]. 
ecancer $2018,12: 889$
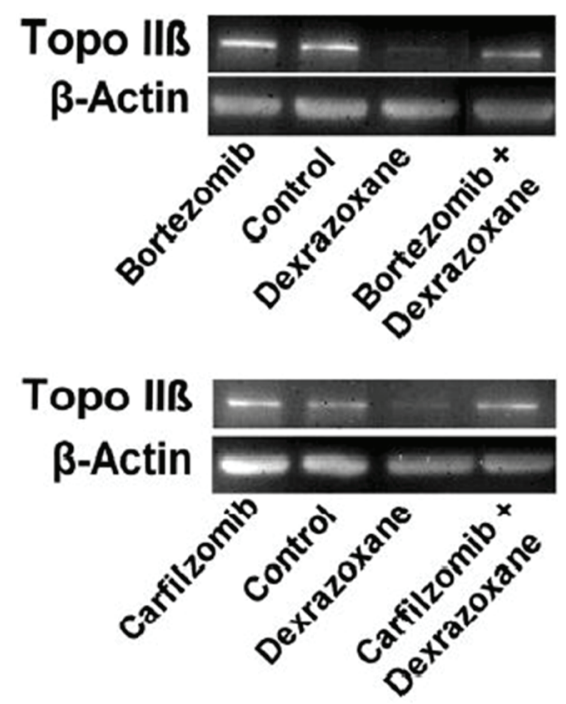

Figure 3. Effect of the proteasome inhibitors, bortezomib and carfilzomib on the dexrazoxane-induced decrease in Top2 $\beta$ levels in neonatal rat cardiomyocytes. Myocytes were treated with bortezomib $(1 \mu \mathrm{M})$ or carfilzomib $(2 \mu \mathrm{M})$ for 30 minutes in growth medium prior to a 6 -hour treatment with dexrazoxane $(100 \mu \mathrm{M})$, lysed and subject to sodium dodecyl sulphate polyacrylamide gel electrophoresis and western blotting. Images reproduced from Hasinoff et al [61] with permission (Springer; Copyright Clearance Licence: 4165441151593).

\section{Introducing the previously-unknown relationship between dexrazoxane and poly(ADP-ribose)}

In 2014, the outcomes of a customised association rule learning algorithm (CEME...Cutting Edge Medical Education), powered to mine the published literature, reveal a previously-unknown relationship between dexrazoxane and poly(ADP-ribose) (PAR) polymer (methods described in [68]).

Historically, the construction of the CEME algorithm was pioneered by McCormack Pharma in the early 1980s from the fundamental tenet whereby a quasi-chaotic relationship exists between experimental data and real-world clinical outcomes. Restated, for a deterministic system that is chaotic, final outcomes are not predictable and are sensitive to starting values. However, in a quasi-chaotic contextdependent system, in common with behaviour in a chaotic system, small differences in starting values likewise result in large differences in final outcomes. However, unlike a true chaotic system, the relationship between the initial conditions and the final outcome is fixed, and accordingly, the experimental data uniquely code for a drug's real-world clinical profile and patterns. In a quasi-chaotic system, CEME displays exquisite sensitivity in probing natural language text for the discovery of new and interesting associations that otherwise would remain lost.

The initial application in 2014 of CEME to dexrazoxane generated 43 outcomes from the published literature that when decoded, resulted in an association rule defining a previously unknown relationship whereby the mechanism of action of dexrazoxane involves some effect upon the synthesis of or an interaction with PAR polymer.

That is, dexrazoxane is a modulator, most likely an inhibitor of either the activity of the polymerase, PAR polymerase (PARP) (most likely PARP1) or PAR polymer function. 


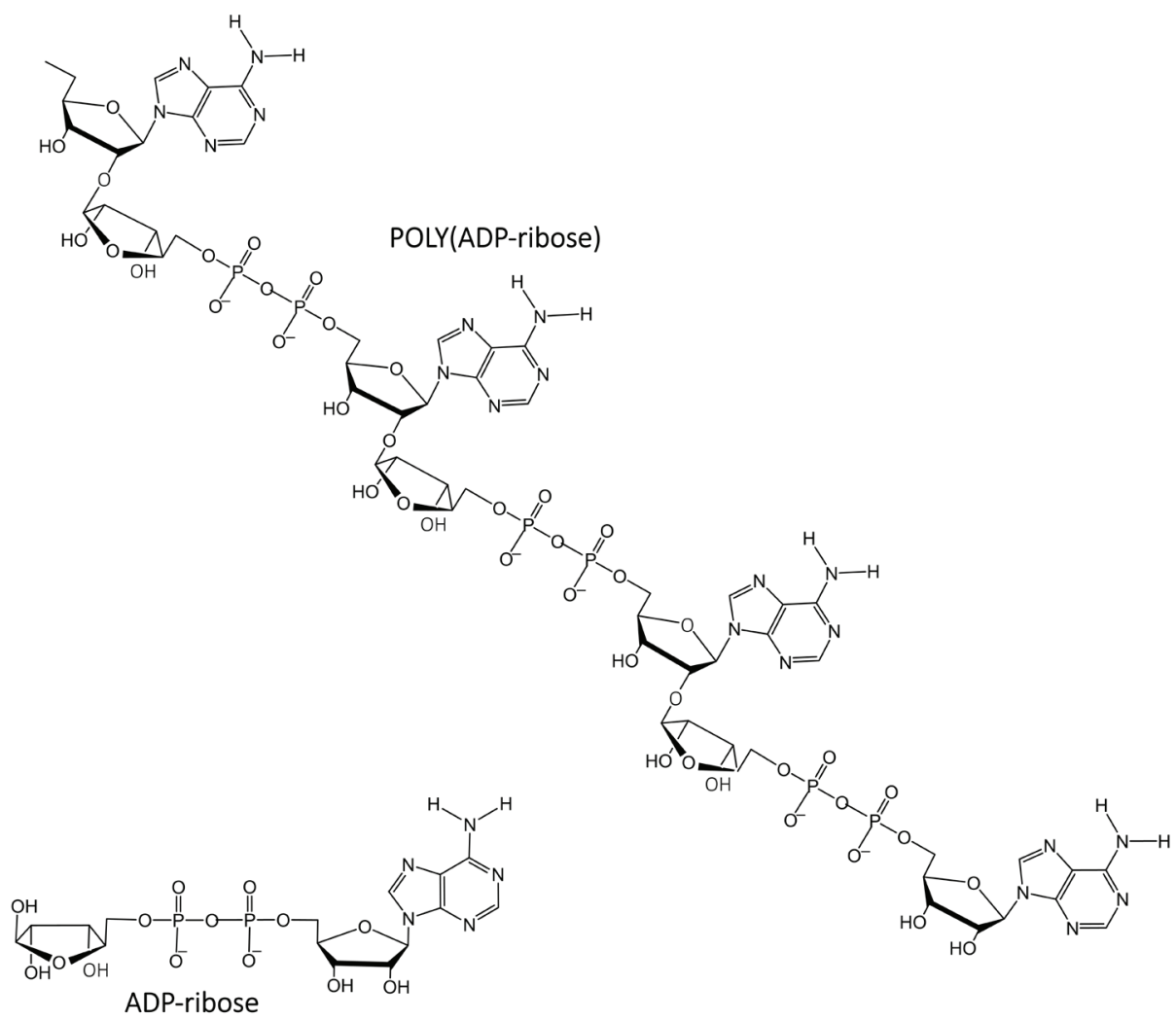

Figure 4. PAR is a polymer of ADP-ribose monomers.

\section{About Poly(ADP-ribose)}

PAR is a polymer of ADP-ribose monomers (Figure 4). PAR synthesis is tightly controlled in mammalian cells with steady-state levels being kept relatively low. However, DNA damage elicits immediate cellular responses that include activation of PARP enzymes with a resultant rapid increase in PAR levels [69]. Among the PARP family members, PARPs 1-3 are DNA damage responsive [70], and functioning as a DNA damage sensor, PARP1 is notable for its well-documented role in orchestrating complex and diverse mechanisms that result in either reparation of DNA lesions or the induction of cell death [71-73].

\section{PARP recognises damaged DNA}

In regulating DNA topology, such as supercoils, the normal catalytic cycle of Top2 $\beta$ consists of a transient enzyme-bridged DNA DSB where the attachment of Top $2 \beta$ to the DNA is via a $5^{\prime}$ tyrosyl phosphodiester covalent linkage. Normally, these transiently cut ends enable relaxation of tangled DNA by a progressive restoration to a less chaotic and lower energy topology. Within the cardiomyocyte, doxorubicin, a Top2 $\beta$ poison targets Top2 $\beta$ and stabilises the Top $2 \beta$-DNA cleavage complex upon the DNA with the result that the DSB is not religated. The effect of this doxorubicin-stabilised Top2 $\beta$-DNA cleavage complex is to create a 'transcriptional roadblock' that arrests elongating RNA polymerase II in its progress to catalyse the transcription of DNA into messenger RNAs. The arrest of RNA polymerase II triggers the ubiquitin/26S proteasome-dependent degradation of Top2 $\beta$. In clearing the stalled transcription machine, the DSB becomes exposed.

PARP1 is one of the first proteins to recognise damaged DNA. In the case of mild DNA damage, the cell activates PARP to facilitate DNA repair. When the levels of DNA damage are beyond the cellular repair capacity, programmed cell death is activated. Extensive DNA damage is accompanied by large-scale PAR polymer synthesis that may lead to a unique form of caspase-independent cell death, 
termed parthanatos (Thanatos from Greek Mythology) whereby PAR polymers translocate to the mitochondria and mediate the release of apoptosis-inducing factor (AIF) from the mitochondria. AIF then translocates to the nucleus and induces cell apoptosis by causing DNA fragmentation and chromatin condensation.

\section{Doxorubicin, PARP and cardiotoxicity}

It has been shown that the activation of PARP contributes to the development of doxorubicin-induced heart failure, and PARP inhibitors have a protective effect [74-76].

Historically, Pacher et al [76] reasoned that activation of PARP may contribute to doxorubicin-induced cardiotoxicity. Using a dual approach of PARP1 suppression, by genetic deletion or pharmacological inhibition with the phenanthridinone-based PARP inhibitor PJ34, these workers demonstrated the role of PARP in the development of cardiac dysfunction induced by doxorubicin. PARP1+/+ and PARP1-/mice received a single injection of doxorubicin. Five days after doxorubicin administration, left ventricular performance was significantly depressed in PARP1+/+ mice, but only to a smaller extent in PARP1-/- mice. Similar experiments were conducted in BALB/c mice treated with PJ34 or vehicle. Treatment with PJ34 significantly improved cardiac dysfunction and increased the survival of the animals. In addition, PJ34 significantly reduced the doxorubicin-induced increase in the serum lactate dehydrogenase and creatine kinase activities, but not metalloproteinase activation in the heart. From these observations, Pacher et al [76] conclude that PARP activation contributes to the cardiotoxicity of doxorubicin. They add that PARP inhibitors may exert protective effects against the development of severe cardiac complications associated with the use of doxorubicin.

Following this early work, the same group investigated the effect of a novel ultrapotent PARP inhibitor, INO-1001 on the development of heart failure induced by permanent ligation of the left anterior descending coronary artery, heart failure induced by doxorubicin and acute myocardial dysfunction induced by bacterial endotoxin [74]. In the coronary ligation model, a significantly depressed left ventricular performance and impaired vascular relaxation of aortic rings were found, and PARP inhibition significantly improved both cardiac function and vascular relaxation. In the doxorubicin model, a single injection of doxorubicin-induced high mortality and a significant decrease in left ventricular systolic pressure, stroke volume, stroke work, ejection fraction and cardiac output. Treatment with the PARP inhibitor reduced doxorubicin-induced mortality and markedly improved cardiac function. Importantly, PARP inhibition did not interfere with doxorubicin's antitumor effect. In the endotoxin model of cardiac dysfunction, PARP inhibition attenuated the suppression of myocardial contractility elicited by endotoxin. Pacher et al [74] conclude that these new data strengthen their earlier view that PARP inhibition may represent an effective approach for the experimental therapy of various forms of acute and chronic heart failure, including that induced by doxorubicin.

Szenczi et al [75] investigated the effects of doxorubicin treatment upon intracellular calcium handling in the hearts of rats after 6 weeks of doxorubicin treatment, under baseline conditions, and in response to oxidative stress induced by hydrogen peroxide exposure in vitro. Following this, they investigated whether pharmacological inhibition of PARP with the PARP inhibitor, PJ34 affected the changes in myocardial mechanical performance and calcium handling in doxorubicin-treated hearts under normal conditions and in response to oxidative stress. Their results showed a marked elevation in intracellular calcium in the doxorubicin-treated hearts which was normalised by pharmacological inhibition of PARP. PARP inhibition also prevented the myocardial contractile disturbances and calcium overload that developed in response to hydrogen peroxide in the doxorubicin-treated hearts. They conclude that their results demonstrate that PARP activation contributes to the development of the disturbances in cellular calcium handling that develop in the myocardium in response to prolonged doxorubicin exposure, and that PARP inhibition beneficially affects chronic morphological and functional alterations in doxorubicin-affected hearts.

Cardiotoxicity studies were undertaken by Ali's group at the School of Pharmacy and Pharmacology, University of Manchester using both male and female CD-1 mice.Consistent with the outcomes from the previous investigators, they observed that the clinically-active PARP inhibitor AG014699 ameliorates doxorubicin-induced cardiotoxicity [77]. They conclude that PARP inhibitors have a role in ameliorating the dose-limiting toxicity of doxorubicin.

\section{Does dexrazoxane target PARP or PAR?}

Following the discovery by the author of a previously unknown relationship between dexrazoxane and PAR (for details of methods see [68]), it remained to determine whether dexrazoxane targets PARP or the polymer product, PAR. Initially, following scrutiny of literature 
reports of pharmacophore identification of compounds targeting PARP1 [78-85], the author concluded that dexrazoxane does not target PARP1. More recent publications [86-88] support this assertion. The overall lack of identity of dexrazoxane with a contemporary pharmacophore of PARP1 is summarised in Figure 5.

\section{In silico modelling reveals an interaction between dexrazoxane and PAR}

If dexrazoxane is not an inhibitor of PARP, then it is not unreasonable to propose that dexrazoxane interacts with PAR. Strikingly, in silico modelling by the author shows that dexrazoxane has the capacity to catalyse the formation of a hybrid self-assembled supramolecular structure between adjacent strands of PAR. Graphically, this assembly depicts an antiparallel orientation of canonical Watson-Crick base pairing of dexrazoxane with adenine bases (Figure 6). That is, within this assembly the non-covalent attractive forces mimic the adeninethymine base-pairing of double-stranded DNA (Figure 7).

- H bonding with Ser243 and Gly202 Yes

- Nicotinamide pharmacophore No

- $\pi-\pi$ interactions with Tyr 246 No

- $\pi-\pi$ interactions with Tyr 228 No

- Low energy conformation within nicotinamide pore No

Figure 5. Lack of identity of dexrazoxane with a contemporary pharmacophore of PARP1.

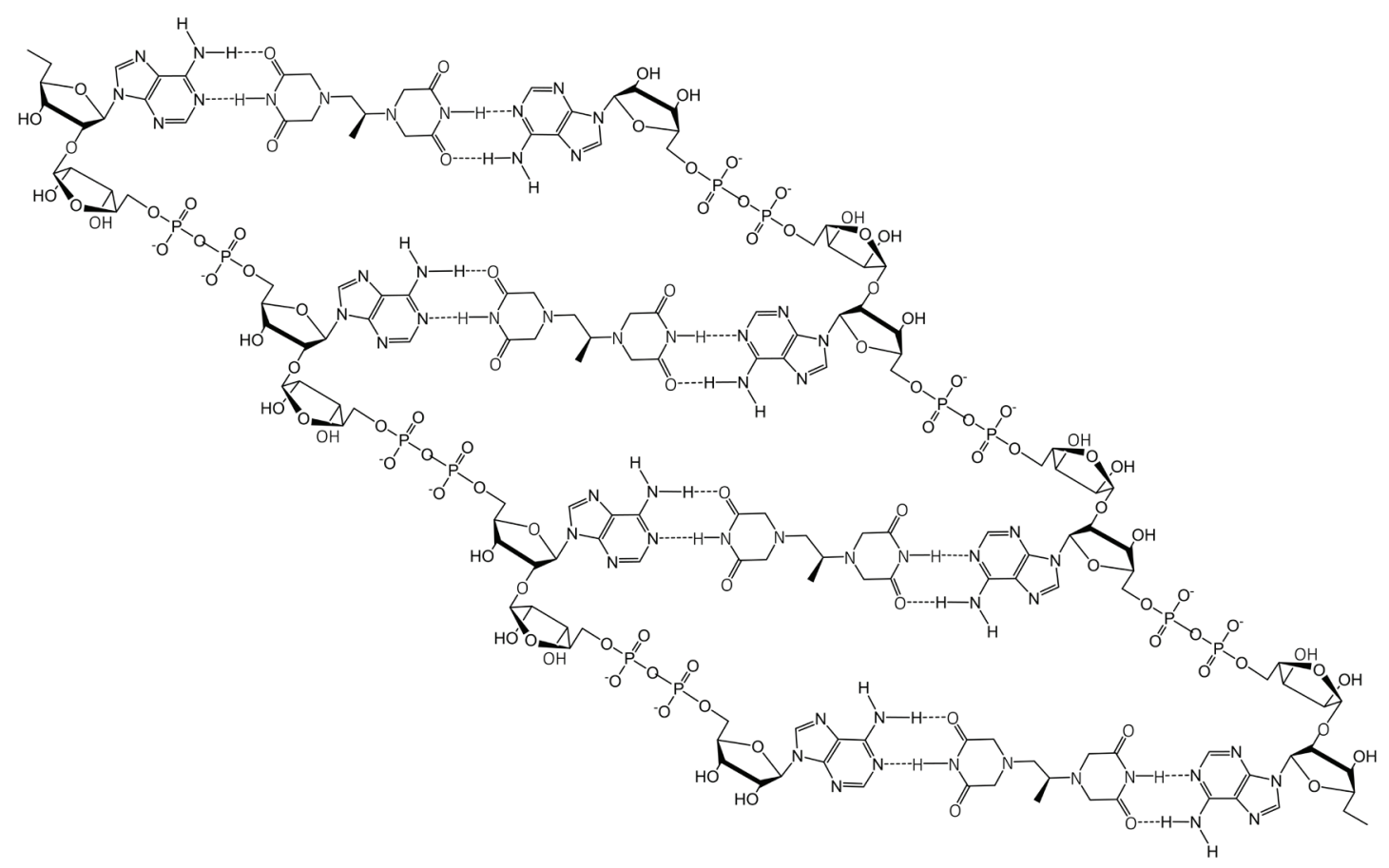

Figure 6. Dexrazoxane catalyses the formation of a hybrid self-assembled supramolecular structure between adjacent strands of PAR. This assembly depicts an antiparallel orientation of canonical Watson-Crick base pairing of dexrazoxane with adenine bases. 


\section{Horizontal forces stabilise the dexrazoxane-PAR self-assembly}

At first inspection, the dexrazoxane-catalysed supramolecular structure with PAR (Figure 6) is distinguished from a DNA duplex by two inseries hydrogen bonded base-pairs at the level of each stack that is directly attributable to the inclusion of dexrazoxane. Indeed, this novel feature is reminiscent of the synthetic fibre, nylon 66 in which hydrogen bonds between adjacent polymer chains result in considerable tensile strength (Figure 8).

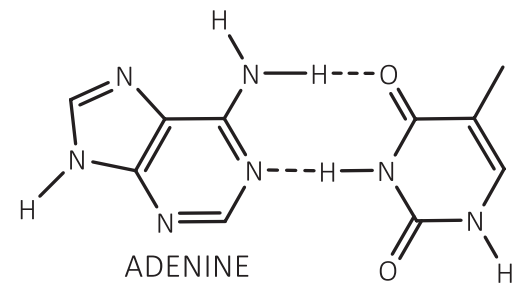

THYMINE

Figure 7. Canonical Watson-Crick adenine-thymine base pairing of double-stranded DNA.

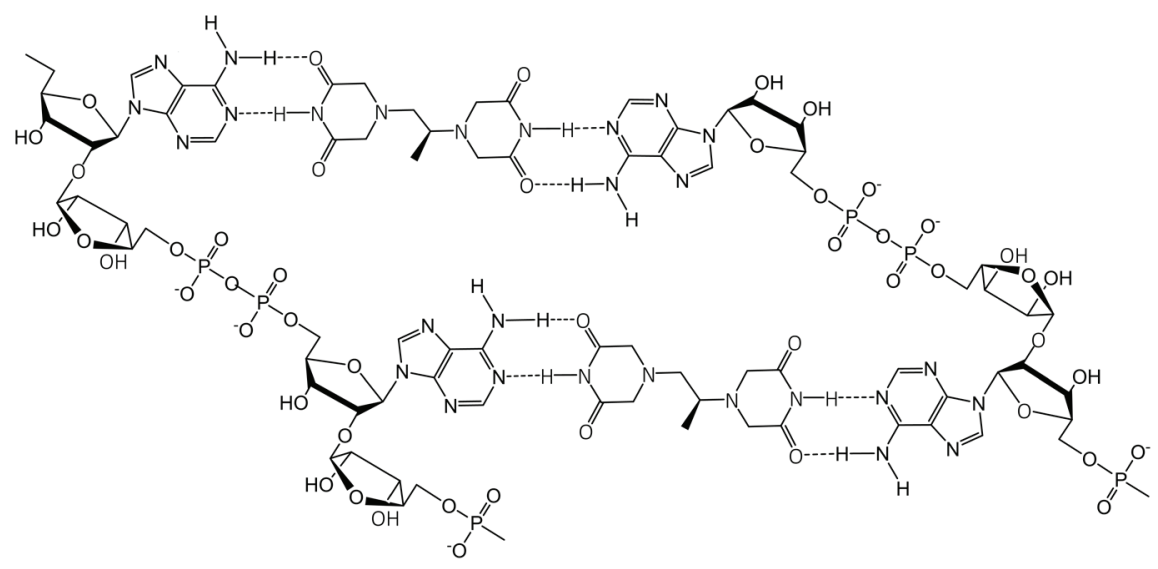

NYLON 66

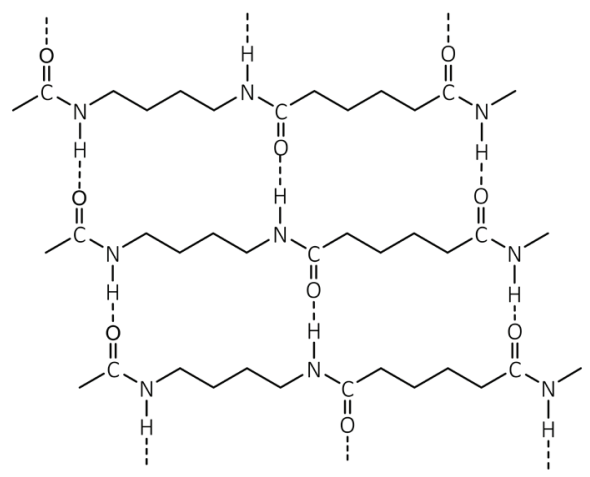

Figure 8. The dexrazoxane-catalysed supramolecular structure with PAR (top diagram) is characterised by two in-series hydrogen bonded base pairs at the level of each stack; this structural feature is reminiscent of the synthetic fibre, nylon 66 in which hydrogen bonds between adjacent polymer chains result in considerable tensile strength. 
The comparison between the dexrazoxane-catalysed supramolecular structure with PAR and the structure of the synthetic fibre, nylon 66 (Figure 8) is significant, not merely because both supramolecular assemblies demonstrate 'in-series' hydrogen bonding, but importantly because the stability of both assemblies is enhanced by an associated, cooperative phenomenon known as the 'Gulliver Principle' [89, 90]. In the same way that the fictitious giant, Gulliver in the land of Lilliput was bound to the ground by a great number of ropes, each of which could be easily broken, together, acting in concert they provided a formidable restraint.

From the outcomes of stability studies with DNA, the increased horizontal distance between PAR strands as a direct result of the interpolation by dexrazoxane (Figures 6 and 8 ) can be anticipated to significantly reduce the repulsive forces between adjacent hydrophilic phosphate groups and minimise destabilising effects of the phosphate-sugar backbone [91, 92].

Conformational studies by Minaga and Kun [93] of long-chain PAR in solution reveal that spectral shifts induced by divalent cations (Mg ${ }^{2+}$ and $\mathrm{Ca}^{2+}$ ) and the highly cationic polyamine, spermine are similar to that reported for DNA. From these seminal observations, Minaga and Kun [93] propose that in common with single-strand DNA, PAR can also adopt a helical conformation and that a double helix cannot be excluded. Importantly, stabilisation of a helical conformation of PAR by increased cationic pressure is entirely consistent with attenuation of the repulsive and destabilising effects of backbone phosphate anions as a direct result of the increased spacing between strands attributable to the inter-strand non-covalent incorporation of dexrazoxane.

In addition, the dexrazoxane-adenine interaction exhibits a dynamic stabilising equilibrium known as tautomerism that thermodynamically favours the keto-amine tautomer (Figure 9). Such mutually reinforcing hydrogen bonds are significantly more stable than regular hydrogen bonds.

By contrast with the horizontally orientated stabilising forces within the PAR-dexrazoxane supramolecular self-assembly, the DNA double helix is stabilised more by vertically orientated base stacking than by horizontal base pairing [91, 94-98]. This statement is consistent with the observation that free bases (adenine, thymine, cytosine and guanine) in water stack on top of one another rather than forming hydrogen-bonded pairs [95, 99-101]. Moreover, vertically orientated stacking interactions are unlikely to contribute to the stabilisation of the hybrid self-assembly of dexrazoxane with PAR given that the distance between the dexrazoxane-adenine stacked assemblies is considerably in excess of $3.4 \AA$ (see below), which is the observed distance between stacked bases in DNA.

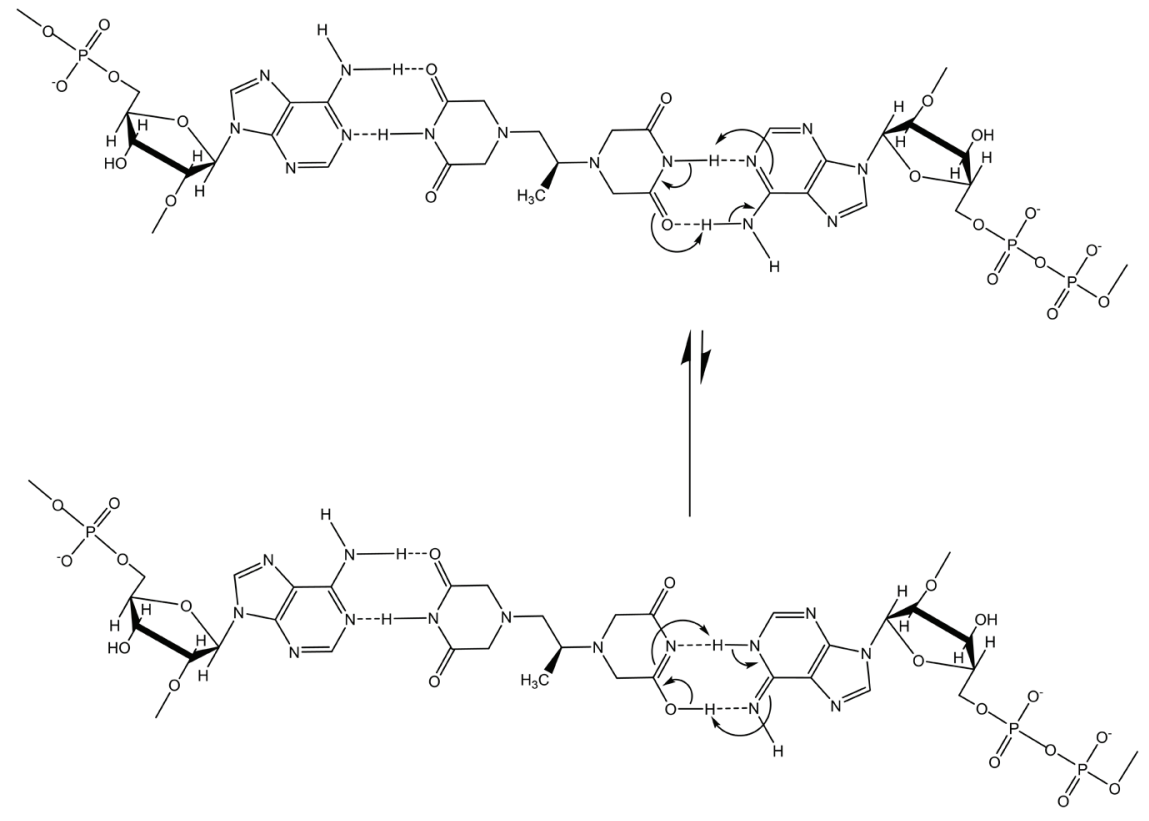

Figure 9. Tautomeric stabilisation of the dexrazoxane-adenine interaction. 
Using a Monte Carlo algorithm, Danilov et al [95] show that at the critical vertical separation of $3.4 \AA$, stacked base dimers (adeninethymine; guanine-cytosine) are energetically favoured by comparison with the corresponding Watson-Crick hydrogen-bonded base pairs. Based upon the results of their simulations they propose that the preference for stacking is due to a significant interaction between each base and water. More recent computer simulations by Mak [91] show that the dominant driving force for stabilising base stacking within DNA is solvent entropy. That is, the simulations show that as the bases get closer to each there is a spontaneous release ('squeezing out') of ordered water molecules from the collective space that they occupy. Mak also demonstrates that stacking is not easily reversed and that at 3.4 $\AA$, separation of stacked bases encounters an energy barrier. This barrier exists because released water cannot easily re-infiltrate the space now occupied by the stacked bases.

Such phenomena, however, cannot be predicted to occur within the PAR-dexrazoxane supramolecular self-assembly with a vertical separation between stacks (as horizontal planes) of approximately 10-12 A. Interestingly, the assembly of the PAR analogue, polyadenine $(\operatorname{poly}(A))$ with the thymine-containing dexrazoxane-like molecule, cyanuric acid likewise appears to depend upon horizontal forces for its robust stability, despite a vertical separation between the adenine-cyanuric acid-adenine stacks that is closer to $3.4 \AA$ (Figure 10 ) $[102,103]$ (discussed in more detail below).

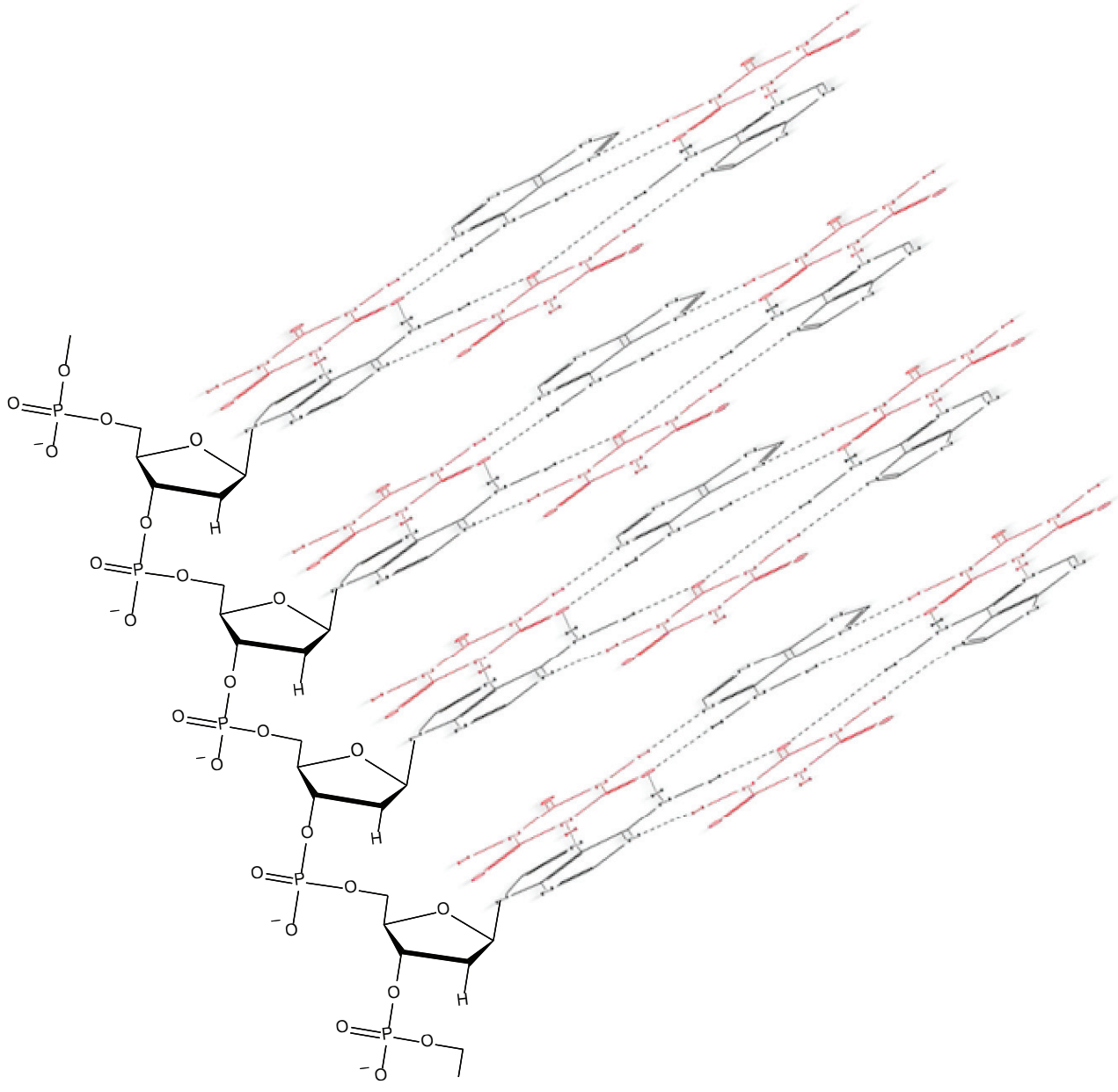

Figure 10. Cyanuric acid (red)-catalysed self-assembly of a poly(A) triplex. Adapted from Avakyan et al [102]. 


\section{Summary}

Key features of the in silico modelling study of the interaction between dexrazoxane and PAR are summarised within Box 1.

Box 1. Key features of the in silico modelling study of the interaction between dexrazoxane and PAR.

- Each of the (bis)dioxopiperazine units of a single molecule of dexrazoxane has the capacity to simultaneously unite the adjacent strands of PAR by a non-covalent interaction with the adenine base of an ADP-ribose moiety of each strand.

- A stack of vertically orientated dexrazoxane molecules between adjacent strands of PAR can be accommodated within a theoretical thermodynamically stable low-energy supramolecular assembly.

- Each repeating subunit of this supramolecular assembly consists of one dexrazoxane molecule and an ADP-ribose moiety from each adjacent strand.

- Within each subunit the non-covalent attractive forces between dexrazoxane and PAR mimic the adenine-thymine base-pairing of double-stranded DNA.

- A thermodynamically stable self-assembly between dexrazoxane and PAR is consistent with a model that depicts an antiparallel orientation of canonical Watson-Crick base-pairing of (bis)dioxopiperazine units with adenine bases.

- The dexrazoxane-catalyzed supramolecular assembly with PAR is distinguished from a DNA duplex by two in-series hydrogenbonded base-pairs at the level of each stack (=subunit) that is directly attributable to the inclusion of dexrazoxane.

- Within supramolecular assemblies, repeating (stacked) in-series hydrogen bonds enhance tensile strength.

- By comparison with a DNA duplex, the increased horizontal distance between PAR strands due to interpolation by dexrazoxane reduces repulsive forces between adjacent hydrophilic phosphate groups that minimizes destabilizing effects of the phosphatesugar backbone.

- The dexrazoxane-adenine interaction exhibits a dynamic stabilizing equilibrium (tautomerism) that thermodynamically favours the keto-amine tautomer; such mutually reinforcing hydrogen bonds are significantly more stable than regular hydrogen bonds.

- The findings herein also indicate that dexrazoxane has the capacity to self-assemble with PAR through a combination of both canonical and non-canonical Hoogsteen base-pairing resulting in the catalysis of a PAR triplex.

- Conformational analysis (see below) confirms that dexrazoxane can align with adenine bases of PAR and form both Watson Crick and Hoogsteen base pairs.

\section{The self-assembly of dexrazoxane with PAR accords with theoretical expectation}

The discovery by the author that dexrazoxane self-assembles with PAR is not surprising. Previously, using an array of compounds, several workers have exploited the non-covalent interaction between adenine, the repeating base of PAR, and thymine (two thymine moieties ('faces') are integral within the (bis)dioxopiperazine moieties of dexrazoxane (Figure 1).

Mixing succinate-modified 2,4,6-triaminopyrimidine, an adenine structural analogue, with cyanuric acid, a thymine structural analogue (Figure 1), Cafferty et al [104] report the formation of extremely long supramolecular assemblies that retain water solubility. In common with the dexrazoxane-PAR assembly, it is the non-covalent interaction of the adenine structures with the thymine faces of cyanuric acid that drives the self-assembly [104].

Of greater relevance to the interaction between dexrazoxane and PAR, however, is the study by Zhou and Bong [105] in which they used a model that incorporated repeating adenine-like structures within a polymer backbone. This study mimics the interaction of dexrazoxane 
with PAR, which, of course, is also a polymer with repeating adenine units. Inspired by Nowick et al [106, 107] who earlier had demonstrated stable adenine-thymine base pairing within aqueous sodium dodecyl sulphate micelles, Zhou and Bong derivatized a polyacrylate polymer strand that incorporated repeating units of the adenine structural analogue, melamine. Using this derivatized polymer, they observed that in an aqueous solution, cyanuric acid triggered the formation of a supramolecular self-assembly consisting of complementary non-covalent interactions between polyacrylate-anchored melamine units and monomeric cyanuric acid (Figure 11).

\section{A parallel model using a close analogue of PAR}

However, while these observations by Zhou and Bong [105] provide insights into the interaction between repeat adenine structures anchored to polymer strands and the thymine faces of a small molecule, it is, however, the more recent work of Sleiman et al [102] that is literally a facsimile of the author's in silico modelling of the interaction between dexrazoxane and PAR. Also, using cyanuric acid, Sleiman et al observed supramolecular self-assemblies with a naturally occurring adenine-containing nucleotide, polyadenine (poly(A)), in both its DNA and RNA forms. Structurally, poly(A) is closely-related to PAR [poly(ADP-ribose)] (Figure 12), and as Figure 1 shows, dexrazoxane and cyanuric acid each contain thymine faces.

Because cyanuric contains three 'thymine faces' (dexrazoxane contains two thymine faces), cyanuric acid was shown by Sleiman et al [102] to self-assemble with three poly(A) strands, as determined by circular dichroism and UV-visible spectroscopy. Cross-sectional plan views that depict the basic units of two different self-assemblies are shown in Figure $13 \mathrm{~A}$ and $\mathrm{B}$. Canonical Watson-Crick base pairing is shown in Figure 13A, and a self-assembly that combines both canonical and non-canonical Hoogsteen base pairing is shown in Figure 13B. Spectral studies by these workers suggest that the hexameric 'rosette' structure, depicted in Figure 13B, is the preferred assembly with cyanuric acid monomers uniting poly $(A)$ strands as a coiling triplex formation. These workers also observed self-assembly using a synthetic adenine-containing peptide nucleic acid in place of poly $(\mathrm{A})$.

A three-dimensional plan view in Figure 10, [for clarity, only a single strand of poly $(A)$ is shown] depicts stacking of the assembly shown in Figure 13B, with the adenine bases (black) mapping the positions of the other two poly $(A)$ strands in the triplex.

As an adjunct to the seminal publication by Sleiman et al [102], further discussion on this pioneering work using poly(A) and cyanuric acid is provided in the review by Berger and Gazit [108]. In addition, in recognising the contribution by Sleiman et al [102], the author wishes to acknowledge a later publication by Tateishi-Karimata and Sugimoto [103] in which these workers also demonstrate the utility of small hydrogen-bonding molecules in orchestrating the self-assembly of nucleic acids in water.

Interestingly, this new report by Sleiman et al [102] provides a novel insight into the author's own work that previously he had not considered, which is that in addition to the self-assembly of two PAR strands by canonical Watson-Crick base pairing (Figure 6), dexrazoxane may also self-assemble with PAR through a combination of both canonical and non-canonical Hoogsteen base pairing to bring together a PAR triplex, as suggested in the cross-sectional plan view in Figure 14. Moreover, the contribution from both type of base pairing may lead to the formation of assemblies beyond a triplex.

In concluding this section, it is both notable and instructive, that the discovery by McCormack Pharma, using an association rule learning algorithm, of an interaction between a nucleic acid and a 'thymine-containing' small molecule predates the publication by Sleiman et al by approximately 2 years [36]. Indeed, that breakthrough discovery by McCormack Pharma achieved initially by mining the existing data resources [36], provided the basis for a subsequent patent application (lapsed) by McCormack and George [109].

\section{Summary}

Key features of published modelling studies of supramolecular self-assemblies using analogues of adenine and thymine or compounds with adenine-like or thymine-like groups in which non-covalent adenine-thymine base-pairing is mimicked are summarised within Box 2. 


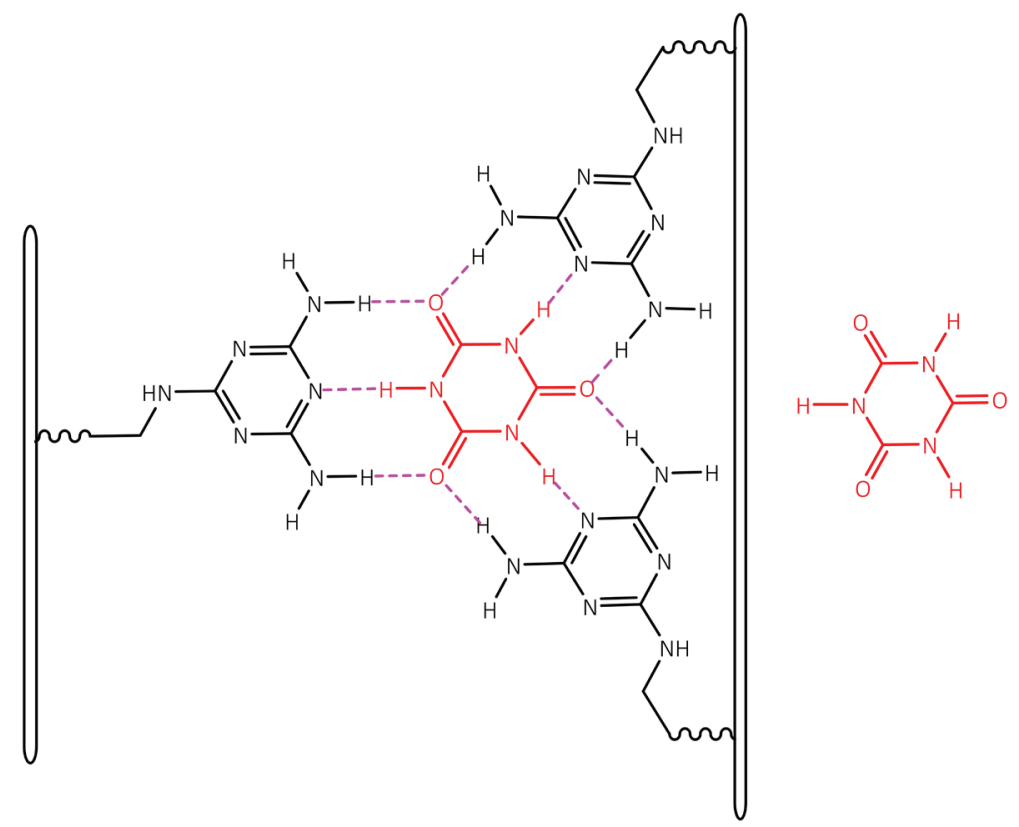

Figure 11. The three thymine faces of cyanuric acid (red) self-assemble with melamine-containing polyacrylate polymer strands. The hydrogenbonding pattern shown is adapted from Zhou and Bong [105].
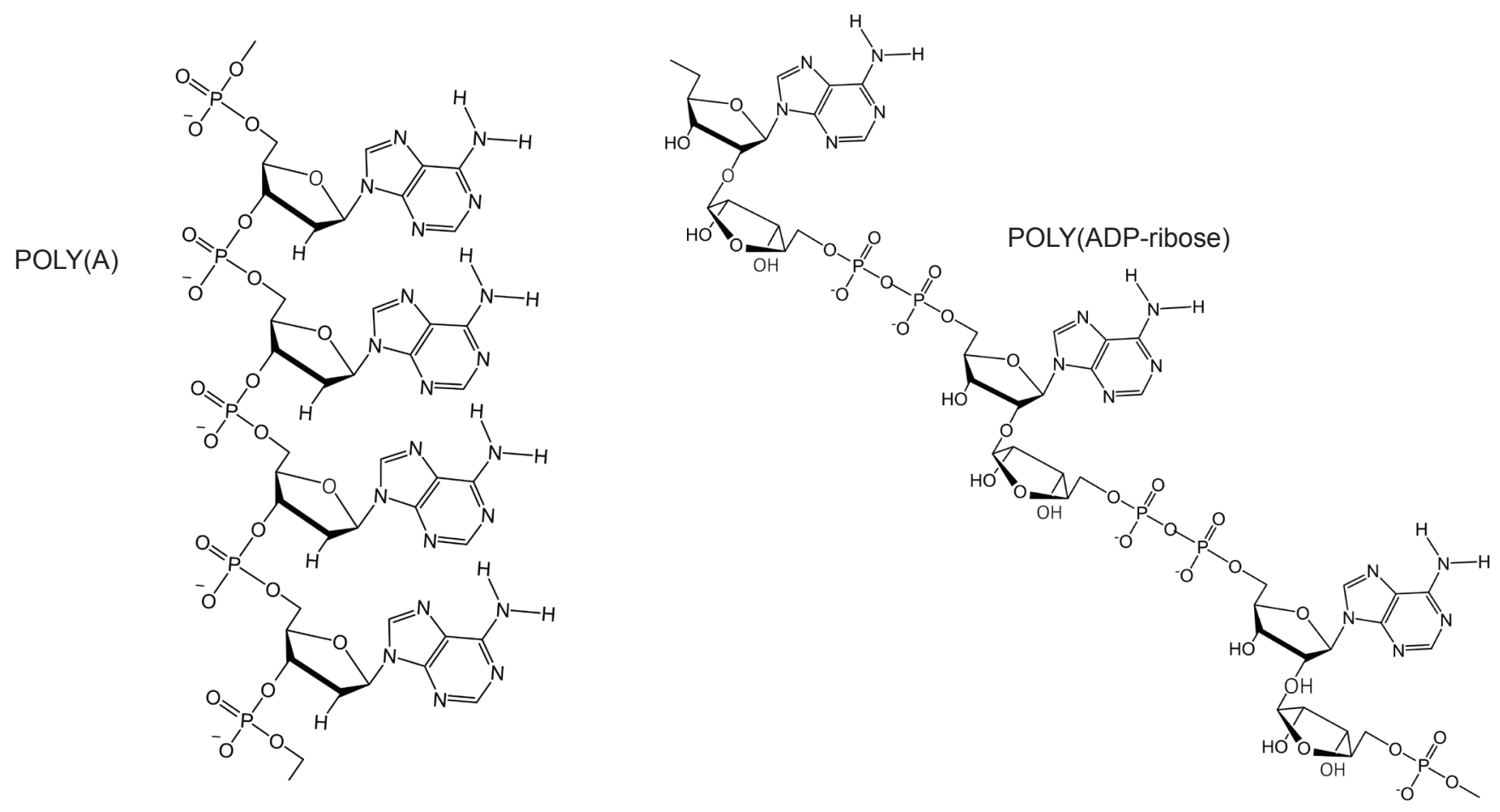

Figure 12. Poly(A) is closely related to PAR [poly(ADP-ribose)]. 

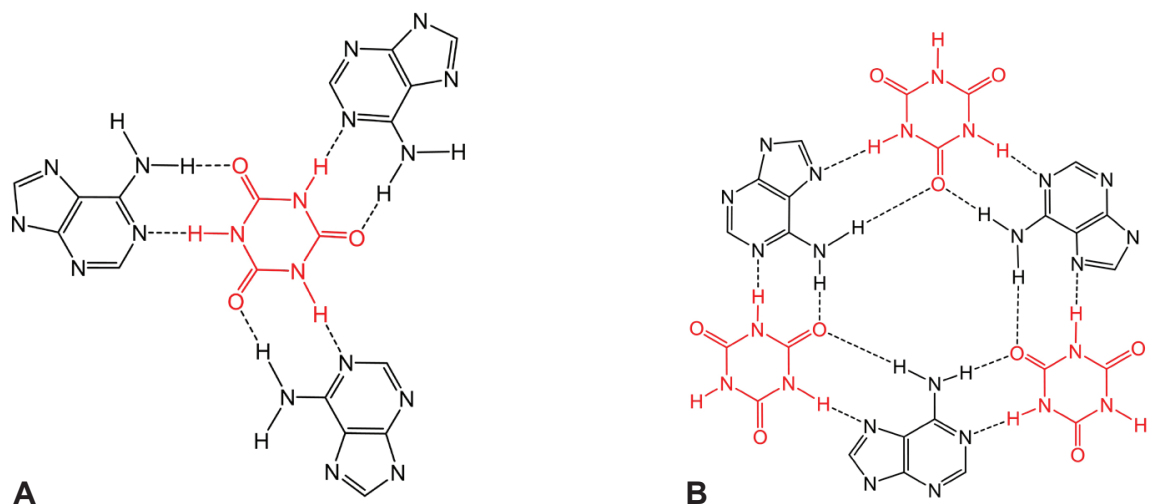

Figure 13. Cyanuric acid monomer(s) self-assembles with three poly(A) strands; canonical Watson-Crick base pairing is shown in (A) and a hexameric rosette structure formed by both canonical and non-canonical Hoogsteen base pairing is shown in B. Adapted from Avakyan et al [102] and Berger and Gazit [108]. (B) adapted with permission (Springer; Copyright Clearance Licence: 4165441151593).

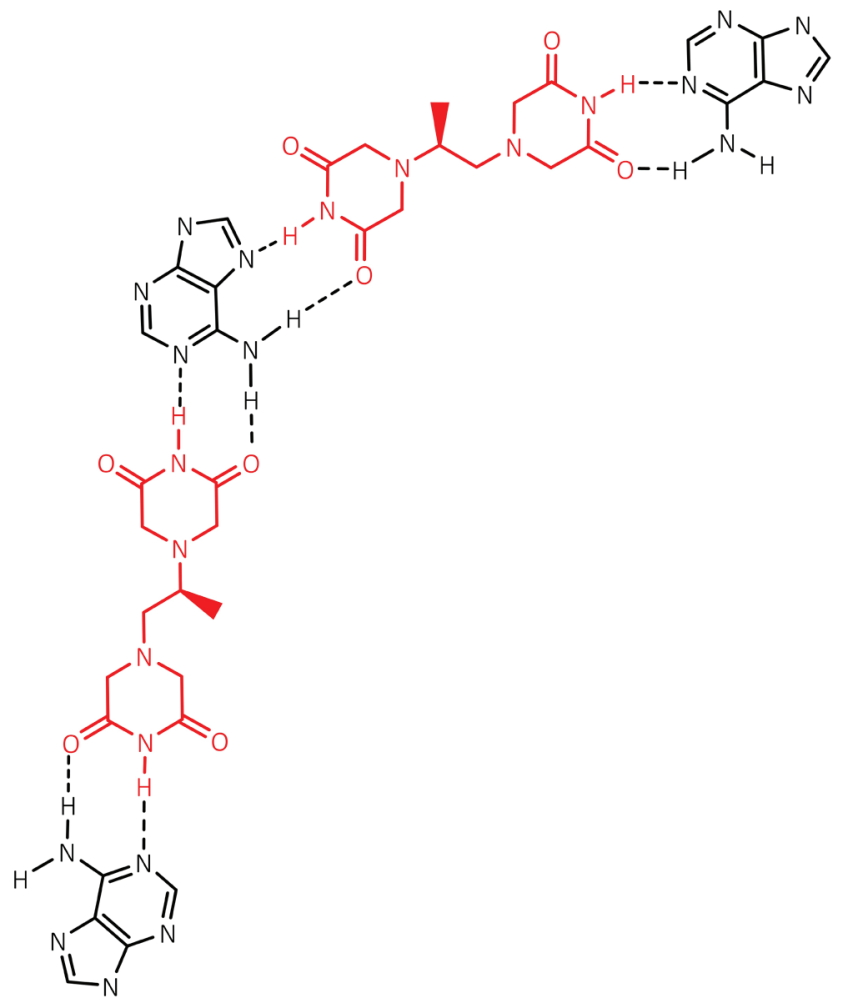

Figure 14. Dexrazoxane may also self-assembly with PAR through a combination of both canonical and non-canonical Hoogsteen base pairing to bring together a PAR triplex; the adenine moieties of PAR are shown in black. Furthermore, the contribution from both types of base pairing may lead to the formation of assemblies beyond a triplex. 
Box 2. Key features of the published modelling studies of supramolecular self-assemblies using analogues of adenine and thymine or compounds with adenine-like or thymine-like groups in which non-covalent adenine-thymine base pairing is mimicked.

- Historically, for an array of compounds, modelling of the adenine-thymine base-pair interaction has been supported by subsequent experimental observations of extensive supramolecular assemblies that are stable in both fully water-solvated systems and aqueous sodium dodecyl sulphate micelles.

- A model incorporating repeating adenine-like structures within a polymer backbone mimics the interaction of dexrazoxane with PAR, a polymer with repeating adenine bases. In aqueous solution, using derivatized polyacrylate polymer strands that incorporate repeating units of the adenine structural analogue melamine, the thymine structural analogue cyanuric acid [a thymine moiety is integral within the each of the (bis)dioxopiperazine units of dexrazoxane] triggered the formation of a supramolecular self-assembly consisting of non-covalent interactions between polyacrylate-anchored melamine units and monomeric cyanuric acid.

- Cyanuric acid catalyses the formation of supramolecular self-assemblies of a naturally occurring adenine-containing nucleotide, polyadenine (poly $(A)$ ), in both its DNA and RNA forms; structurally, poly $(A)$ is closely related to PAR. Cyanuric acid-catalysed self-assemblies of poly $(A)$ can be modelled using either canonical Watson-Crick base pairing or a combination of both canonical Watson-Crick and non-canonical Hoogsteen base pairing. Spectral studies suggest that the preferred assembly of poly(A) strands with cyanuric acid monomers is a coiling triplex formation consisting of both canonical and non-canonical base pairing.

- Cyanuric acid-catalysed self-assembly using a synthetic adenine-containing peptide nucleic acid (PNA) in place of poly(A) has also been demonstrated.

\section{Conformational analysis confirms that dexrazoxane aligns with adenine bases}

Intuitively, it is to be expected that low-energy conformations will exist when there is a maximum separation between the piperazine structures. Conformational analysis undertaken by the author (for details of methods see the appended Supplement) confirm that lower energy conformations are consistent with the maximum spacing between piperazine structures with each adopting a half-chair conformation at methylene carbon atoms 3,5 of each ring structure resulting in an elevated tertiary amine nitrogen atom. As a half-chair conformation, the remainder of the piperazine structure is planar with the keto group at the carbon atom in position 2 of each ring structure in alignment with the tautomeric nitrogen of the secondary amine group of the adenine base of each ADP-ribose unit of PAR (using the orientation depicted in Figure 6). This analysis confirms that dexrazoxane can align with adenine bases of PAR and form both Watson-Crick and Hoogsteen base pairs.

\section{Self-assembly enables a deep compartment for the accumulation of dexrazoxane}

A model can be constructed that demonstrates the self-assembly process of dexrazoxane with PAR (Figure 15). For clarity, while Figure 15 depicts the self-assembly occurring at the termini of long-chain PAR strands (each strand can have a length in excess of 300 ADP-ribose units), dexrazoxane can interact with PAR at any part of the strand. Dynamically, the model illustrates how the anthracycline-compromised cardiomyocyte represents a deep compartment for the accumulation of dexrazoxane. Uncharged dexrazoxane transits the lipid membrane of the cardiomyocyte and encounters PARP-elaborated PAR within the intracellular compartment.

\section{Dexarazoxane selectively accumulates within doxorubicin-compromised cardiomyocytes}

Dexrazoxane in assembly with PAR (Figure 15) is effectively trapped resulting in a concentration gradient of dexrazoxane into the intracellular compartment. Upon inspection, this model is clearly critically concentration sensitive. That is, in an in vitro simulation, high concentrations 
of dexrazoxane at early times above a critical concentration of dexrazoxane will saturate PAR strands and sterically hinder free molecules of dexrazoxane from binding simultaneously, but not necessarily coincidentally, with two strands of PAR. However, in vivo, it is likely that the concentration of PAR, especially at early times, is greatly in excess of the concentration of dexrazoxane with the result that free molecules of dexrazoxane can non-covalently bind with two strands of PAR. As discussed above (Figure 14), a combination of canonical and non-canonical Hoogsteen base-pairing may result in an interaction between dexrazoxane and PAR strands that is more complex than that illustrated in Figure 15.

\section{Dexrazoxane prevents AIF release from isolated mitochondria}

In collaboration with Ritchie et al (Unpublished observations, Dr Kenneth J Ritchie, School of Pharmacy and Biomolecular Sciences, Liverpool John Moore's University, Liverpool UK, Dec 2015), a pilot study was undertaken by the author to investigate the interaction between dexrazoxane and PAR. Using isolated rat liver mitochondria, we demonstrated for the first time that dexrazoxane inhibits PAR-induced release of AIF. Although the details of this methodology are not presented here, the western blots from this study, using anti-AIF antibody (Abcam ab32516) are reproduced in Figure 16.

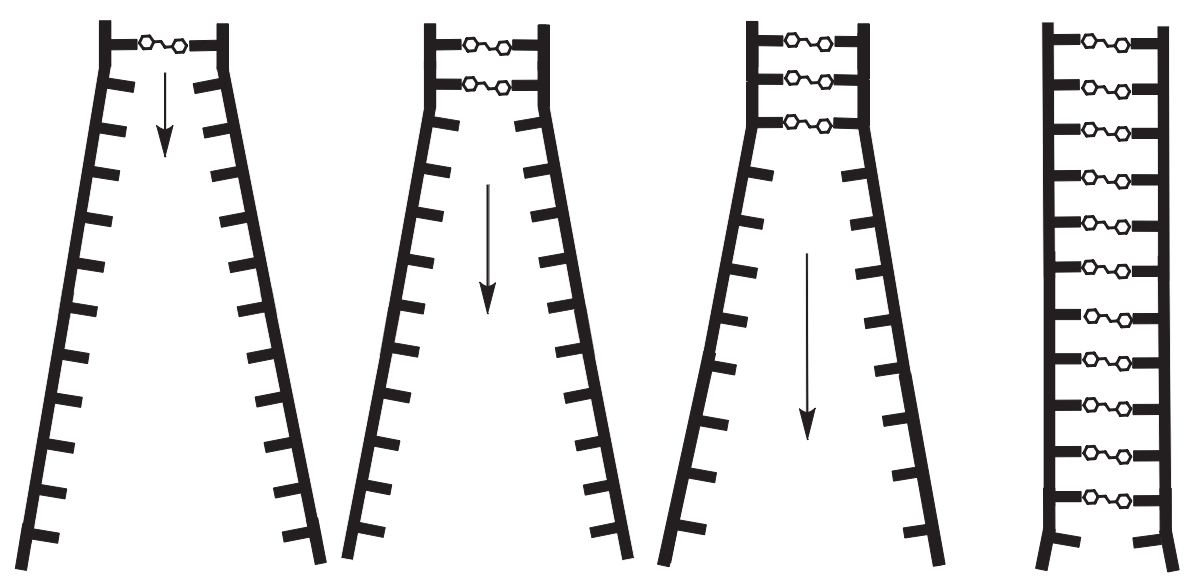

Figure 15. The anthracycline-compromised cardiomyocyte represents a deep compartment for the accumulation of dexrazoxane. Uncharged dexrazoxane transits the lipid membrane of the cardiomyocyte and encounters PARP-elaborated PAR within the intracellular compartment.

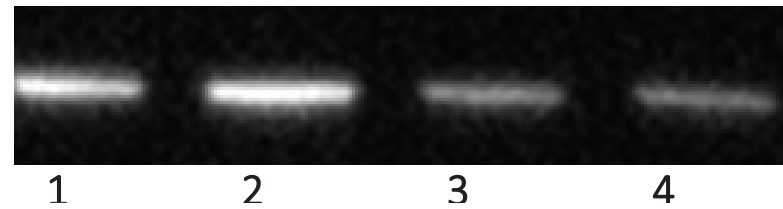

Figure 16. Dexrazoxane prevents AIF release from isolated mitochondria. Track 1 = Control; track $2=100 \mathrm{nM}$ PAR and $100 \mathrm{nM}$ dexrazoxane; track 3 = $100 \mathrm{nM}$ PAR and $50 \mathrm{nM}$ dexrazoxane; track 4 = $100 \mathrm{nM}$ PAR and $10 \mathrm{nM}$ dexrazoxane. PAR and dexrazoxane were incubated together for 10 minutes before adding to the mitochondrial suspension (western blots reproduced from the study final report). 
Interestingly, and initially somewhat surprisingly, under the conditions of this preliminary study, we observed an inverse relationship between the concentration of dexrazoxane and the release of AIF from isolated mitochondria. With hindsight, this observation is consistent with a saturating concentration of dexrazoxane that within the short equilibrium period (10 minutes) binds extensively and randomly to individual PAR strands, thereby precluding organised and thermodynamically favoured self-assembly between dexrazoxane and PAR strands (Figure 15). Dose ranging and equilibrium times require further evaluation; notably, the parallel paradigm by Sleiman et al [102] using poly(A), a polymer closely related to PAR used an equilibration time of 12 hours.

Importantly, combining dexrazoxane with PAR in vitro is an inadequate paradigm of the in vivo deep compartment of the anthracyclinecompromised cardiomyocyte described above. Further studies should incorporate the use of whole cells, such as isolated neonatal rat cardiomyocytes. The use of ionising radiation to induce DNA damage with the subsequent elaboration of PAR would enable a more exact study of the interaction between dexrazoxane and PAR with minimal confounding ([111], Personal communication Dr Kenny Ritchie Dec 2015). In this way, the lipid boundary imposed by the cell membrane will preclude saturation of PAR by dexrazoxane.

\section{Concluding remarks}

Scientific truth is a moving target [112], and as the eminent Austrian Ethologist and Nobel Laureate, Konrad Lorenz famously remarked 'Truth in science can be defined as the working hypothesis best suited to open the way to the next better one' [114].

Conceptually, all research consists of three basic components: data, conclusions and hypotheses. The not inconsiderable controversy about the truth of published research findings (claims) is elegantly argued by loannidis as being critically dependent upon the robustness of the conclusions [113]. The author of this review shares the view of loannidis [113], echoed by the remarks of Lorenz [114] that all too frequently most research conclusions are false. However, it is inescapable that in many scientific fields research claims will always include a component from prevailing bias. Consequently, that most conclusions are false is an inevitable part of the research endeavour and, accordingly, conclusions will always be subject to change; approaching the truth but never arriving. Accepting this (and I concede that many will not), it is the responsibility of scientific and clinical investigators to embrace uncertainty (and many do).

In this review, the author examines the burgeoning hypothesis that dexrazoxane protects the heart against anthracycline-induced damage by promoting the proteasomal degradation of Top $2 \beta$, the Top2 isoenzyme that predominates in cardiomyocytes. This hypothesis has its origins within the guiding tenet that Top $2 \beta$ is required to initiate the entire phenotypic cascade of doxorubicin-induced cardiomyopathy. Consequently, it can be reasonably concluded that if doxorubicin is introduced into a system that is devoid of Top2 $\beta$, then the effects of doxorubicin that are mediated by Top2 $\beta$ will not happen. This is a safe conclusion.

Several studies show that treatment with dexrazoxane results in the coincident depletion of Top2 $\beta$ protein. Taken together, we may conclude that the effects of both dexrazoxane and doxorubicin converge upon Top $2 \beta$. This is a safe conclusion. Beyond this, no conclusions upon the relationship between Top2 $\beta$ and the cardioprotective mechanism of dexrazoxane are safe. Collectively, the above conclusions are 'hypothesis generating' and nothing more.

In the current examination of the 'Top2 $\beta$ hypothesis', at the outset, throughout and before arriving at any kind of conclusion, the author formulated key questions in an endeavour to reduce prevailing bias that otherwise might influence his own conclusions. Failing to do this and relying solely upon scrutiny and pooling of published 'effect sizes' will achieve little else other than reproducing the net bias that is inherent in the existing published claims. Such key questions include, but were not limited to:

- How many other compounds deplete levels of Top2 $\beta$ protein? Do compounds that deplete levels of Top2 $\beta$ protein protect the heart from the effects of anthracyclines? Indeed, it must be iterated that there is a consensus that the doxorubicin-stabilised Top2 $\beta$-DNA cleavage complex creates a 'transcriptional roadblock' that arrests elongating RNA polymerase II; the arrest of RNA polymerase II triggers the ubiquitin/26S proteasome-dependent degradation of Top2 $\beta$. In isolation, this observation raises alarm bells alerting us to the message that the depletion of Top $2 \beta$ protein is likely a ubiquitous phenomenon that has more to do with the proteasomalmediated clearance of Top $2 \beta$ protein transcriptional roadblocks, and little if anything to do with the cardioprotective mechanism of a single drug. 
- Is the cardioprotective efficiency of dexrazoxane compromised when the level of Top2 $\beta$ protein is manipulated (sustained), by blocking the ubiquitin/26S proteasome machine for example? The findings from the erudite study by Hasinoff et al [61] using dexrazoxane in combination with a proteasome inhibitor are provocative and demand a reappraisal of the earlier conclusions from other groups.

- What is known about the stoichiometry between doxorubicin, Top2 $\beta$ protein and cardiomyocyte toxicity? The seminal contributions by Kersting et al [57] and Lipshultz et al [60] permit the extrapolation by the author that in the heart near-zero levels of Top2 $\beta$ protein are a sufficient and functional target for doxorubicin. At best, the in vivo results show that the limited lowering of Top2 $\beta$ protein level by dexrazoxane is transient and short lived (hours) within a setting of a concomitant rapid recovery by newly synthesised Top $2 \beta$ protein.

- What is known about the relative mean residence times of the simultaneous, but not coincident administration of dexrazoxane and doxorubicin in clinical practise? Within the context of persistently elevated levels of doxorubicin over a period of days and weeks, it is difficult to understand how in a clinical setting, lowering of Top $2 \beta$ protein levels following a single 15-minute infusion of dexrazoxane with a mean half-life of 2.2 hours [34] can exert a cardioprotective effect.

\section{A different approach}

In 2014, in the author's hands, the outcomes of a customised association rule learning algorithm incorporating the use of antecedent surrogate variables (CEME, McCormack Pharma [68]) reveal a previously unknown relationship between PAR polymer.

Historically, CEME was conceived by the author's Group in the early 1980s and has its origins in the fundamental tenet whereby a quasichaotic relationship exists between experimental data and real-world clinical outcomes.

Quasi-chaotic systems are characterised by a fixed relationship between initial conditions and the final outcome. Accordingly, experimental data uniquely code for a drug's real-world clinical profile and patterns. In a quasi-chaotic system, CEME displays exquisite sensitivity in probing natural language text for the discovery of new and interesting associations that otherwise would remain lost.

The initial application in 2014 of CEME to dexrazoxane revealed an unequivocal signal defining a previously-unknown relationship between dexrazoxane and PAR polymer. Subsequently, the unique property of dexrazoxane to sequester PAR by base pairing was demonstrated using in silico modelling and a preliminary in vitro study confirmed an interaction. This previously-unknown relationship explains both acute and long-term cardioprotection in patients receiving anthracyclines. In addition, as an inhibitor of PAR dexrazoxane has access to the epigenome and this offers a new insight into protection by dexrazoxane against doxorubicin-induced late-onset damage [McCormack K, manuscript in preparation]. Notably, echocardiographic data suggest that dexrazoxane provides long-term cardioprotection, implying that prevention of cardiomyocyte damage during therapy can reduce the incidence of delayed doxorubicin-associated cardiomyopathy in longterm survivors without compromising the chances of oncological cure [117, 118].

What does the proposed association between dexrazoxane and PAR mean to Oncologists?

In addressing this important question some historical perspective must be introduced. Dexrazoxane reduces the incidence of anthracyclineinduced heart failure by $80 \%$ and it is the only drug approved for its prevention [161]. Despite this impressive clinical effect, the use of dexrazoxane is somewhat limited largely because of historical concerns of interference with the antitumor efficacy of anthracyclines [157], induction of secondary malignancies and myelodysplastic syndrome [158]. However, subsequent meta-analyses do not support these concerns [159]. Moreover, prolonged survival of doxorubicin responders co-treated with dexrazoxane has been reported [160].

While beyond the scope of this review, the clinical consensus is that dexrazoxane does not compromise anti-tumour therapy. Indeed, in May 2017, the EMA Committee for Medicinal Products for Human Use approved the expansion of the Cardioxane Marketing Authorisation enabling increased use of dexrazoxane within the paediatric population for whom dexrazoxane had been previously contraindicated (dexrazoxane is marketed by Clinigen UK as a branded generic). A review of the evidence that historically led to a reassessment of the European Label for dexrazoxane found that dexrazoxane is not associated with an increased risk of second primary malignancies and that dexrazoxane therapy does not impair anthracycline's anti-tumour efficacy [119]. 
Thus, if the above changes and revisions lead to an increase in the use of dexrazoxane throughout different patient populations, then the alert clinician should be responsive to novel/atypical/unexpected clinical benefits and outcomes if dexrazoxane is acting in some part by sequestering PAR by base-pairing.

Indeed, scrutiny of existing clinical outcomes supports an interaction between dexrazoxane and PAR. For example, Lipshultz et al propose that impaired cardiac function in doxorubicin-treated childhood cancer survivors is partly mediated by disruption of mitochondrial energy production and that this damage is abrogated by dexrazoxane [60]. In a long-term, cross-sectional study, these investigators examined mitochondrial DNA (mtDNA) copy numbers per cell and oxidative phosphorylation (OXPHOS) in PBMCs in 64 childhood survivors of highrisk ALL. Importantly, these workers argue that changes within PBMC mitochondria reflect what is occurring in cardiomyocyte mitochondria.

At a median follow-up of 7.8 years after treatment, doxorubicin-treated survivors without dexrazoxane had increased PBMC mtDNA copies per cell and concomitant use of dexrazoxane was associated with lower mtDNA copies per cell. The investigators add that mtDNA copies per cell in those who received dexrazoxane were within the normal ranges observed in their previous studies. OXPHOS activity was not different between groups. The investigators propose that in patients treated with doxorubicin alone, impaired mitochondria may undergo clonal expansion of mtDNA that functionally compensates for mutations or deletions that results in normal OXYPHOS and ATP production within the myocardium. They add that their findings support the evidence that dexrazoxane adjuvant therapy in paediatric high-risk ALL patients offers systemic mitochondrial protection and suggest a possible role of dexrazoxane prior to anthracycline therapy as a general protectant of mitochondrial function in other healthy tissues that include the ovaries [122].

Previously, Lebrecht et al [120] had proposed that mtDNA alterations initiated during acute doxorubicin exposure persist and accumulate during the long term and that this process may represent an important factor in the delayed onset of anthracycline-associated cardiomyopathy; they cite work that indicates some mtDNA mutations may persist for a long time because of inefficient repair [121].

Notwithstanding alternative explanations, can the above effects upon mtDNA be reconciled with an interaction between dexrazoxane and PAR, and in the absence of dexrazoxane can PAR explain the delayed onset of anthracycline-associated cardiomyopathy?

Using wild-type and PARP1-depleted A549 cells, Szczesny et al [123] investigated the role of PARP1 in the repair of mtDNA under oxidative stress conditions. They showed that intra-mitochondrial PARP1 interacts with mitochondrial-specific DNA base excision repair enzymes, endonuclease G-like 1 (EXOG) and DNA polymerase gamma (Poly), which under oxidative stress become PARylated (a post-translational modification whereby PAR polymers are covalently-attached to proteins by PARPs). Notably, they observed that the presence of PARP1 adversely affected the integrity of mtDNA which was attributable to a pronounced inhibition of the repair of mtDNA. By contrast, repair of oxidative-induced damage to the mitochondrial DNA in PARP1-depleted cells was found to be more robust, and by comparison with the wild-type cells, this was associated with an enhancement of mitochondrial biogenesis. Szczesny et al speculate that by contrast with the well-established positive regulatory role of PARP1 in maintaining nuclear DNA integrity, the addition of PAR groups on mtDNA repair enzymes may uniquely reduce their affinity to bind with DNA and thus may attenuate the rate of mtDNA repair.

Accepting the new hypothesis that dexrazoxane sequesters PAR by base pairing, then arguably this sequestration state is comparable/ equivalent to the PARP1-depleted cells used by Szczesny et al [123] and that sequestration of PAR by dexrazoxane can be envisaged to compromise the complex PARylation process [124]. For example, sequestration of PAR by dexrazoxane may disrupt the alignment of the highly negatively charged backbone of PAR polymers and accordingly disrupt electrostatic interactions between the PARylated substrate and proteins. In addition, sequestration of PAR may influence recognition of the PARylated substrate by so-called 'ADP-ribose readers' that contain ADP-ribose/PAR-binding domains [124]. Accordingly, the abrogation of impaired mitochondrial function by dexrazoxane reported by Lipshultz et al [60] may be attributable in part to an attenuation by dexrazoxane of the potential inhibitory effects of PARylation upon mtDNA repair following exposure to anthracycline therapy [120].

A further example serves to illustrate how the existing data can be re-evaluated in the light of an interaction between dexrazoxane and PAR. While the acute cardiotoxic effects of doxorubicin are consistent with a doxorubicin-induced DNA damage response resulting in both PARP1-dependent apoptotic and necrotic cell death [125, 126], delayed-onset doxorubicin-induced cardiomyopathy that may become manifest years or decades following the initial administration of doxorubicin, in addition to protracted effects upon the integrity of mtDNA 
$[60,120,123]$ is also consistent with epigenetic alterations that induce a pathological shift in the relative expression of myosin heavy chain $(\mathrm{MHC})$ isoforms $[135,152]$.

Two isoforms of $\mathrm{MHC}$ are expressed in the mammalian heart, $\alpha-\mathrm{MHC}$ and $\beta-\mathrm{MHC}$. The $\alpha-\mathrm{MHC}$ isoform has a higher ATPase activity than $\beta-\mathrm{MHC}$, and the contractile velocity of the heart is correlated with the relative amount of each isoform; hearts expressing greater amounts of $\alpha-\mathrm{MHC}$ having a more rapid contractile velocity [136-138].

The $\alpha$ - and $\beta-\mathrm{MHC}$ ratio correlates directly with the overall cardiac performance in both animals and in patients with cardiomyopathy and heart failure [139-145]. Pathological hypertrophy of adult hearts is associated with $\alpha-\mathrm{MHC}$ downregulation and $\beta-\mathrm{MHC}$ induction, which is a relationship that characterises the foetal phenotype of MHC expression [146]. However, hearts expressing $\alpha-\mathrm{MHC}$ have better outcomes under stress conditions than those expressing mainly $\beta-\mathrm{MHC}[139,140,142]$. Consequently, strategies that prevent or reverse undesirable shifts in the relative proportions of $\mathrm{MHC}$ isoforms represent attractive and fundamental approaches in the management of cardiomyopathies [147].

De Beer's Group in the Department of Medical Physiology at Utrecht University observed that long-term administration of doxorubicin resulted in an impairment of the actin-myosin interaction in the hearts of male Wistar rats [148]. Notably, assays of ventricular muscle revealed a shift from the adult expressing high-ATPase isoform, $\alpha-\mathrm{MHC}$, to the foetal low-ATPase isoform, $\beta$-MHC. From these results and that of an earlier study by the same group [149], these workers conclude that the direct effect of chronic treatment with doxorubicin upon the actin-myosin system provides an additional mechanism through which anthracyclines exert their cardiotoxic effects.

Following their earlier observations of the effects of chronic treatment with doxorubicin upon the contractile function of the heart, de Beer et al investigated whether the cardio-protective properties of dexrazoxane involve the prevention of the deleterious effects of doxorubicin upon the actin-myosin contractile machinery [150]. They observed that dexrazoxane treatment offered significant protection against the doxorubicin-induced impairment of kinetics; time constants describing transitions between different states in the actin-myosin interaction were restored to normal when trabeculae were exposed to sudden changes in length in the so-called 'quick release' and 'slack-test'. Tissue from both right and left ventricles was examined for MHC content using gel electrophoresis, and laser scanning densitometry was used to identify differences in myosin isoform composition. Whereas doxorubicin treatment significantly increased the $\beta-\mathrm{MHC}$ to $\alpha-\mathrm{MHC}$ ratio, notably, dexrazoxane prevented the shift from the high ATPase $\alpha-\mathrm{MHC}$ isoform towards the low ATPase $\beta$-MHC isoform.

Interestingly, there are no reports within the literature of an association between Top2 $\beta$ and MHC isoforms, and additional studies by de Beer et al do not support a role for free radicals in mediating the deleterious effects of doxorubicin upon the actin-mysoin contractile machinery [150]. However, delayed-onset toxicity associated with exposure to doxorubicin, may be explained by a phenotypic switch that results from doxorubicin-induced alteration(s) at the epigenome [McCormack K, manuscript in preparation]. Recently, Ferreira and coworkers using male Wistar rats, demonstrated that doxorubicin modulates gene expression patterns via alterations to the epigenetic landscape, notably the DNA methylome in the heart but not liver and that such changes contribute to doxorubicin-induced delayed-onset cardiomyopathy [151]. Moreover, disrupted cardiac mitochondrial biogenesis, as demonstrated by decreased mtDNA levels (see above) was also observed.

Are doxorubicin-induced alterations at the epigenome as manifest by a shift in the ratio between MHC isoforms and the effects of dexrazoxane in abrogating this shift, consistent with an interaction between dexrazoxane and PAR?

Hang et al in the Division of Cardiovascular Medicine at Stanford University School of Medicine show that Brahma-related gene 1 (Brg1), a chromatin-remodelling ATPase protein subunit of the BRG-1-Associated Factor (BAF complex), interacts with two other classes of chromatin-modifying enzymes, histone deacetylase (HDAC) and PARP, to regulate gene expression during cardiac growth, differentiation and hypertrophy [152]. In adults, Brg1 is turned off in cardiomyocytes. However, it is re-activated by cardiac stresses and Brg1 complexes with its embryonic partners, HDAC and PARP, to induce a pathological shift from $\alpha-\mathrm{MHC}$ to $\beta-\mathrm{MHC}$ expression. In addition to HDACs, PARP1 is the only other chromatin-modifying enzyme known to regulate cardiac hypertrophy [153-155]. In addition, Hang et al show that in hypertrophic hearts PARP1 is bound to the proximal promoters of $\alpha-M H C$ and $\beta-M H C$, and that inhibition of PARP1 activity by the phenanthridinonebased PARP inhibitor PJ34 reduced both Brg1-mediated $\alpha-M H C$ repression and $\beta-M H C$ activation in reporter assays, indicating that Brg1 and PARP1 cooperate to regulate MHC expression [152]. 
Notably, Mahesh Gupta's Group in the Department of Cardiothoracic Surgery at The University of Chicago demonstrate that nuclear extracts treated with anti-ADP-ribose antibodies reveal increased poly-ADP-ribosylation (PARylation) of nuclear proteins in failing hearts by comparison with controls, thus confirming the presence of PAR polymer [156]. From this observation, they propose that post-translational modification of proteins by PAR interferes with their ability to bind to each other and to DNA, resulting in repression of gene transcription and finally cell death. Attenuation of cardiac hypertrophy in $\mathrm{PARP}^{-/-}$mice was also evident from the analysis of hypertrophic marker genes. In $\mathrm{PARP}^{+/+}$aortic artery-banded mice, the levels of $\beta-\mathrm{MHC}$ mRNA were highly elevated, whereas $\alpha-\mathrm{MHC}$ levels were repressed, as expected. By contrast, in PARP ${ }^{-/}$aortic artery-banded mice, no repression of $\alpha-\mathrm{MHC}$ levels was observed, and the levels of $\beta$-MHC were increased to a much lesser extent.

In their concluding remarks, they propose that PARP inhibitors may be useful agents for managing cardiomyopathies and heart failure.

Taken together, these and other data are provocative and indicate that in some part, the cardiotoxic effects of doxorubicin and the cardioprotective effects of dexrazoxane, converge upon the epigenome. By comparison with acute damage, delayed-onset toxicity is consistent with a phenotypic switch that results from doxorubicin alteration(s) at the epigenome. Sequestration of PAR through both canonical Watson-Crick base pairing, and non-canonical Hoogsteen base-pairing provides a mechanism for the cardioprotective effects of dexrazoxane against the toxic effects of doxorubicin in the acute term, and abrogation of delayed-onset cardiomyopathy.

Given the focal role of PAR in an overwhelming number of diseases, it is indeed thought provoking that dexrazoxane offers, for the first time, a unique opportunity to investigate the impact of sequestration of PAR, by comparison with inhibition of PARP, within a remarkably broad context. By way of example, and whilst beyond the scope of this review, the interaction between dexrazoxane and PAR offers the prospect that dexrazoxane can function as a cytoprotectant within other physiological systems (Lipshultz et al have previously suggested a possible role of dexrazoxane prior to anthracycline therapy as a general protectant of mitochondrial function in healthy tissues in addition to the heart [60]). Neuroprotection offered by dexrazoxane in a preclinical model of neurological dysfunction outperformed that of established neuroprotectants that included a blocker of the N-methyl-D-aspartate receptor channel, dizolcipline (MK-801), a calcium channel blocker, nimodipine, and an iron chelator, deferoxamine (desferal), resulting in significantly greater survival rates and attenuated neurological deficits [115]. In that study, Rodriguez et al [115] conclude that dexrazoxane has 'very important neuroprotective properties'.

\section{Limitations}

The CEME algorithm is a well-validated methodology that has been successfully applied by McCormack Pharma to many drugs throughout more than three decades [68, 127-134, 187]. CEME is a process that discovers 'previously unknown relationships' within the published literature for a nominated drug within an elected therapeutic category. By comparison with the kind of data stored in structured databases, natural language text within the published literature is unstructured, amorphous and difficult to mine using the traditional algorithms. In practise, the greatest obstacle is missing data within a dataset that is small (thousands of data pieces) by comparison with the much larger size (millions of data pieces) of dataset sets mined using methods traditionally applied in commercial operations such as mining high street supermarket transactions. CEME overcomes the problem of missing data by adapting the use of a statistical method known as 'antecedent surrogate variables'. Specifically, in exploring the mechanism of action of dexrazoxane as a cardioprotectant, the task was to characterise as exhaustively as possible cell signalling within all known molecular phenotypes (as determined by extracellular first messengers) of anthracycline-exposed cardiomyocytes. In practise, this is an extremely demanding operation that requires the manual input for all known molecular phenotypes of all known second messengers, enzymes, receptors, transcription factors and so on. To reduce the possibility of a type I error (false positive), in addition to dexrazoxane, the CEME process should be repeated for all known cardioprotectants of anthracycline-exposed cardiomyoctes; the list includes beta blockers, angiotensin inhibitors, angiotensin-converting enzyme inhibitors and statins. That is, previously unknown relationships between each cardioprotectant and cell signalling within the anthracycline-compromised cardiomyocyte should be explored. For practical reasons, this was not undertaken.

In silico modelling studies demonstrate that dexrazoxane catalyses the formation of a hybrid self-assembled supramolecular structure between adjacent strands of PAR. These assemblies depict an antiparallel orientation of canonical Watson-Crick base pairing of dexrazoxane with adenine bases. In addition, these in silico studies show that dexrazoxane can also base pair with PAR strands by noncanonical Hoogsteen base pairing. Importantly, these supramolecular structures employing either canonical or non-canonical base pairing 
and combinations of the two, accord with the theoretical expectation of low-energy thermodynamically stable entities. Moreover, that such supramolecular assemblies are possible is strongly suggested by the work of other groups using molecules that are closely analogous to both dexrazoxane and PAR [102, 104-107]. However, confirmation that the supramolecular assemblies between dexrazoxane and PAR are stable under in vivo conditions is lacking. Initially, aqueous stability could be explored by utilising in vitro methods such as thermal denaturation using circular dichroism and ultraviolet-visible spectroscopy [102]. These critically necessary studies remain to be undertaken.

The results of a preliminary in vitro study show that consistent with expectation, dexrazoxane prevents PAR-induced AIF release from isolated mitochondria. However, dose-ranging studies are needed, and equilibrium times require investigation. The utilisation of exogenous PAR as used by the author is not a paradigm of a deep compartment for the accumulation of dexrazoxane and consequently does not adequately represent the in vivo situation. Preferably, further studies should incorporate the use of whole cells, such as isolated neonatal rat cardiomyocytes together with the use of ionising radiation to induce DNA damage with subsequent elaboration of endogenous PAR.

\section{Supplementary materials and methods}

The conformational relationship of the non-covalent interaction between dexrazoxane and PAR was explored using a modified version of Allinger's Molecular Mechanics MM2 force field (for review see [110]). Accordingly, conformational analysis with a dihedral driver was performed and minimum energy conformations were computed for dexrazoxane using Chem3D 16.0 with a minimum root mean squared gradient set to 0.1 . Semi-empirical molecular orbital calculations were also undertaken using the Chem3D 16.0 tool MOPAC in order to eliminate unstable high energy conformers and for geometry optimisation. Conformational analysis and energy minimisation was additionally supported by the Chem3D 16.0 tool, CONFLEX (CONFLEX Corporation). Dissociation constants as pKa that were not evident within the literature were calculated using methods provided within Chemaxon (MarvinSketch).

\section{Acknowledgments and conflicts of interest}

The association rule mining, in silico modelling, and preclinical investigations were supported by a grant from Clinigen Healthcare Ltd., Burton-On-Trent, UK. The author is the first inventor on a lapsed patent application: McCormack K and George PL (2015) PAR Inhibition. Patent Application Number PCT/GB2015/054026; Publication number WO2016051213 A1.

The author gratefully acknowledges the erudite discussions during preparation of the manuscript with Peter L George FIBMS MBA MSc, and the meticulous efforts by Dr Kenneth J Ritchie at Liverpool John Moores University in the investigation of the effects of dexrazoxane upon PAR-induced release of AIF from isolated mitochondria.

\section{References}

1. Zhang S, Liu X, and Bawa-Khalfe T, et al (2012) Identification of the molecular basis of doxorubicin-induced cardiotoxicity Nat Med 18 1639-1642 https://doi.org/10.1038/nm.2919 PMID: 23104132

2. Vejpongsa P and Yeh ET (2014) Topoisomerase 2 $\beta$ : a promising molecular target for primary prevention of anthracyclineinduced cardiotoxicity Clin Pharmacol Ther 95 45-52 https://doi.org/10.1038/clpt.2013.201

3. Moudgil R and Yeh ET (2016) Mechanisms of cardiotoxicity of cancer chemotherapeutic agents: cardiomyopathy and beyond Can J Cardio/ 32 863-870 https://doi.org/10.1016/j.cjca.2016.01.027 PMID: $\underline{27117975}$ PMCID: 4921299

4. Lyu YL, Kerrigan JE, and Lin C-P, et al (2007) Topoisomerase IIB-mediated DNA double-strand breaks: implications in doxorubicin cardiotoxicity and prevention by dexrazoxane Cancer Res 67 8839-8846 https://doi.org/10.1158/0008-5472.CAN-07-1649 PMID: 17875725 
5. Yeh ETH (2014) Topoisomerase $\mathbf{2 b}$ as a predictor of susceptibility to anthracycline-induced cardiotoxicity United States Patent Application Publication Application Number 14/155,858 Publication Number US 2014/0200192 A1

6. Xiao H, Mao Y, and Desai SD, et al (2003) The topoisomerase llbeta circular clamp arrests transcription and signals a 26S proteasome pathway Proc Natl Acad Sci USA 100 3239-3244 https://doi.org/10.1073/pnas.0736401100 PMID: 12629207 PMCID: $\underline{152276}$

7. Mao Y, Desai SD, and Ting CY, et al (2001) 26S proteasome-mediated degradation of topoisomerase II cleavable complexes J Biol Chem 276 40652-40658 https://doi.org/10.1074/jbc.M104009200 PMID: 11546768

8. Xiao H and Goodrich DW (2005) The retinoblastoma tumor suppressor protein is required for efficient processing and repair of trapped Topoisomerase II-DNA cleavable complexes Oncogene 24 8105-8113 https://doi.org/10.1038/sj.onc.1208958 PMID: 16091739 PMCID: 2799250

9. Yeh ETH (2018) Doxorubicin-induced cardiotoxicity: the role of topoisomerase $2 \beta$ (Bethesda, MD: National Institute of Health)

10. van Asperen J, van Tellingen $O$, and Tijssen F, et al (1999) Increased accumulation of doxorubicin and doxorubicinol in cardiac tissue of mice lacking mdr1a P-glycoprotein Br J Cancer 79 108-113 https://doi.org/10.1038/sj.bjc.6690019 PMID: 10408701 PMCID: 2362153

11. Nwankwoala R, Georgewill O, and Georgewill U (2008) Pharmacokinetics of adriamycin after intravenous administration in rat Intern J Oncol 64

12. Rahman A, Carmichael D, and Harris M, et al (1986) Comparative pharmacokinetics of free doxorubicin and doxorubicin entrapped in cardiolipin liposomes Cancer Res 46 2295-2299 PMID: $\underline{3697976}$

13. Ozols RF, Locker GY, and Doroshow JH, et al (1979) Pharmacokinetics of adriamycin and tissue penetration in murine ovarian cancer Cancer Res 39 3209-3214 PMID: $\underline{455305}$

14. Staples BJ, Pitt WG, and Roeder BL, et al (2010) Distribution of doxorubicin in rats undergoing ultrasonic drug delivery J Pharm Sci 99 3122-3131 https://doi.org/10.1002/jps.22088 PMID: 20166203 PMCID: 4533826

15. Chenard P (2015) A comparison of doxorubicin and doxorubicinol in rat heart and liver tissue following anthracycline administration MSc Thesis The Faculty of Graduate Studies at Laurentian University, Sudbury, Ontario, Canada

16. Amin AM, Lowe MC, and Ismail AA (1983) Cytofluorescence localization of adriamycin-binding sites of cardiac tissue Oncology 40 340-343 https://doi.org/10.1159/000225758 PMID: $\underline{621996}$

17. Stewart DJ, Grewaal D, and Green RM, et al (1993) Concentrations of doxorubicin and its metabolites in human autopsy heart and other tissues Anticancer Res 13 1945-1952 PMID: 8297100

18. Prescribing information FDA label (2014) Doxorubicin Hydrochloride for Injection Distributed by Pfizer Laboratories, Division of Pfizer Inc, New York, NY 10017, USA

19. Goormaghtigh E, Chatelain P, and Caspers J, et al (1980) Evidence of a complex between adriamycin derivatives and cardiolipin: possible role in cardiotoxicity Biochem Pharmacol 29 3003-3010 https://doi.org/10.1016/0006-2952(80)90050-7 PMID: 7458950

20. Rockley KL, Richardson G, and Gill JH (2016) Characterisation of novel molecular mechanisms involved in anthracyclineinduced cardiotoxicity Poster presentation Safety Pharmacology Society meeting Vancouver, Canada

21. Zhao $L$ and Zhang $B$ (2017) Doxorubicin induces cardiotoxicity through upregulation of death receptors mediated apoptosis in cardiomyocytes Sci Rep 744735 https://doi.org/10.1038/srep44735 PMID: 28300219 PMCID: 5353581

22. Wei L, Wang SF, and Gu HW, et al (2017) A new impedimetric detection of cardiotoxicity induced by doxorubicin in cultured neonatal rat cardiomyocytes Biomed Res 28 199-202 
23. Ncardia (2018) Detection of cardiac-specificty of anthracyclines using ES Cell-derived Cor At cardiomyocytes Unpublished, Ncardia USA, Wayne PA 19087, USA [www.ncardia.com]

24. Junkun L, Erfu C, and Hasahya T, et al (2016) Curcumin downregulates phosphate carrier and protects against doxorubicin induced cardiomyocyte apoptosis Biomed Res Int 2016(1) 1-6 https://doi.org/10.1155/2016/1980763

25. Tawagi $\mathrm{E}$, Massmann $\mathrm{C}$, and Chibli $\mathrm{H}$, et al (2015) Differential toxicity of gold-doxorubicin in cancer cells vs. cardiomyocytes as measured by real-time growth assays and fluorescence lifetime imaging microscopy (FLIM) Analyst $1405732-5741$ https://doi. org/10.1039/C5AN00446B PMID: 26161455

26. Lamberti M, Porto S, and Marra M, et al (2012) 5-Fluorouracil induces apoptosis in rat cardiocytes through intracellular oxidative stress Exp Clin Cancer Res 3160 https://doi.org/10.1186/1756-9966-31-60

27. Menna P, Salvatorelli E, and Minotti GJ (2007) Doxorubicin degradation in cardiomyocytes Pharmacol Exp Ther $322408-419$ https:// doi.org/10.1124/jpet.107.122820

28. Inoue T, Tanaka K, and Mishima M, et al (2007) Predictive in vitro cardiotoxicity and hepatotoxicity screening system using neonatal rat heart cells and rat hepatocytes Proc 6th World Congress on Alternatives \& Animal Use in the Life Sciences (August 21-25, Tokyo, Japan) AATEX Special Issue 14 457-462

29. Hasinoff BB and Aoyama RG (1999) Relative plasma levels of the cardioprotective drug dexrazoxane and its two active ringopened metabolites in the rat Drug Metab Dispos 27 265-268 PMID: 9929514

30. Hasinoff BB (1994) An HPLC and spectrophotometric study of the hydrolysis of ICRF-187 (dexrazoxane, (+)-1,2-bis(3,5dioxopiperazinyl-1-yl)propane) and its one-ring opened intermediates Int J Pharm 107 67-76 https://doi.org/10.1016/0378$\underline{5173(94) 90303-4}$

31. Hasinoff BB (1994) Pharmacodynamics of the hydrolysis-activation of the cardioprotective agent (+)-1,2-bis(3,5-dioxopiperazinyl-1-yl)propane J Pharma Sci 83 64-67 https://doi.org/10.1002/jps.2600830115

32. Hasinoff BB (1990) The hydrolysis activation of the doxorubicin cardioprotective agent ICRF-187 [+)-1,2-bis(3,5-dioxopiperazinyl-1-yl)propane) Drug Metab Dispos 18 344-349 PMID: 1974197

33. Schroeder PE, Jensen PB, and Sehested M, et al (2003) Metabolism of dexrazoxane (ICRF-187) used as a rescue agent in cancer patients treated with high-dose etoposide Cancer Chemother Pharmacol 52 167-174 https://doi.org/10.1007/s00280-003-0619-7 PMID: 12750840

34. Prescribing information Summary of Product Characteristics (2018) CARDIOXANE 500 mg powder for solution for infusion Marketing Authorisation holder Clinigen Healthcare Ltd, Burton-on Trent, Staffordshire, DE14 2WW, UK

35. Earhart RH, Tutsch KD, and Koeller JM, et al (1982) Pharmacokinetics of (+)-1,2-di(3,5-dioxopiperazin-1-yl)propane intravenous infusions in adult cancer patients Cancer Res 42 5255-5261 PMID: $\underline{614754}$

36. McCormack K (2014) Use of a customised association rule learning algorithm incorporating the application of antecedent surrogate variables to determine the mechanism of action of dexrazoxane Unpublished

37. Hasinoff BB and Herman EH (2007) Dexrazoxane: how it works in cardiac and tumor cells. Is it a prodrug or is it a drug? Cardiovasc Toxicol 7 140-144 https://doi.org/10.1007/s12012-007-0023-3 PMID: 17652819

38. Hasinoff BB, Schroeder PE, and Patel D (2003) The metabolites of the cardioprotective drug dexrazoxane do not protect myocytes from doxorubicin-induced cytotoxicity Mol Pharmacol 64 670-678 https://doi.org/10.1124/mol.64.3.670 PMID: 12920203

39. Hasinoff BB, Takeda K, and Ferrans VJ, et al (2002) The doxorubicin cardioprotective agent dexrazoxane (ICRF-187) induces endopolyploidy in rat neonatal myocytes through inhibition of DNA topoisomerase II Anticancer Drugs 13 255-258 https://doi. org/10.1097/00001813-200203000-00007 PMID: 11984069 
40. Hasinoff BB, Kuschak TI, and Yalowich JC, et al (1995) A QSAR study comparing the cytotoxicity and DNA topoisomerase II inhibitory effects of bisdioxopiperazine analogs of ICRF-187 (dexrazoxane) Biochem Pharmacol 50 953-958 https://doi. org/10.1016/0006-2952(95)00218-O PMID: $\underline{7575679}$

41. Yan T, Deng S, and Metzger A, et al (2009) Topoisomerase II\{alpha\}-dependent and -independent apoptotic effects of dexrazoxane and doxorubicin Mol Cancer Ther 8 1075-1085 https://doi.org/10.1158/1535-7163.MCT-09-0139 PMID: 19417146

42. Deng S, Yan T, and Jendrny C, et al (2014) Dexrazoxane may prevent doxorubicin-induced DNA damage via depleting both topoisomerase II isoforms BMC Cancer 14842 https://doi.org/10.1186/1471-2407-14-842 PMID: 25406834 PMCID: 4242484

43. Lin RK, Ho CW, and Liu LF, et al (2013) Topoisomerase II deficiency enhances camptothecin-induced apoptosis Biol Chem 288 7182-7192 https://doi.org/10.1074/jbc.M112.415471

44. Lee KC, Padget $\mathrm{K}$, and Curtis $\mathrm{H}$, et al (2012) MRE11 facilitates the removal of human topoisomerase Il complexes from genomic DNA Biol Open 1 863-873 https://doi.org/10.1242/bio.20121834 PMID: 23213480 PMCID: 3507232

45. Kawatani M, Takayama $\mathrm{H}$, and Muroi M, et al Identification of a small-molecule inhibitor of DNA topoisomerase II by proteomic profiling Chem Biol 18 743-751 PMID: 21700210

46. Sunter NJ, Cowell IG, and Willmore E, et al (2010) Role of topoisomerase liß in DNA damage response following IR and etoposide J Nucleic Acids 2010 pii: 710589 https://doi.org/10.4061/2010/710589

47. Van Hille B, Perrin D, and Hill BT (1999) Differential in vitro interactions of a series of clinically useful topisomerase-interacting compounds with the cleavage/relegation activity of the human topoisomerase llalpha and llbeta isoforms Anticancer Drugs 10 551-560 https://doi.org/10.1097/00001813-199907000-00007

48. Alchanati I, Teicher C, and Cohen G, et al (2009) The E3 ubiquitin-ligase Bmi1/Ring1A controls the proteasomal degradation of Top2alpha cleavage complex-a potentially new drug target PLoS One 4 e8104 https://doi.org/10.1371/journal.pone.0008104

49. Ban Y, Ho CW, and Lin RK, et al (2013) Activation of a novel ubiquitin-independent proteasome pathway when RNA polymerase II encounters a protein roadblock Mol Cell Biol 33 4008-4016 https://doi.org/10.1128/MCB.00403-13 PMID: 23938298 PMCID: $\underline{3811683}$

50. Azarova AM, Lyu YL, and Lin CP, et al (2007) Roles of DNA topoisomerase II isozymes in chemotherapy and secondary malignancies Proc Natl Acad Sci U S A 104 11014-11019 https://doi.org/10.1073/pnas.0704002104 PMID: 17578914 PMCID: 1904155

51. Zhang A, Lyu YL, and Lin CP, et al (2006) A protease pathway for the repair of topoisomerase II-DNA covalent complexes $J$ Biol Chem 281 35997-36003 https://doi.org/10.1074/jbc.M604149200 PMID: 16973621

52. Azarova AM, Lin RK, and Tsai YC, et al (2010) Genistein induces topoisomerase llbeta- and proteasome-mediated DNA sequence rearrangements: implications in infant leukemia Biochem Biophys Res Commun 399 66-71 https://doi.org/10.1016/j. bbrc.2010.07.043 PMID: 20638367 PMCID: 3376163

53. Isik S, Sano K, and Tsutsui K, et al (2003) The SUMO pathway is required for selective degradation of DNA topoisomerase Ilbeta induced by a catalytic inhibitor ICRF-193(1) FEBS Lett 546 374-378 https://doi.org/10.1016/S0014-5793(03)00637-9 PMID: 12832072

54. Barthelmes HU, Niederberger E, and Roth T, et al (2001) Lycobetaine acts as a selective topoisomerase II beta poison and inhibits the growth of human tumour cells Br J Cancer 85 1585-1591 https://doi.org/10.1054/bjoc.2001.2142 PMID: 11720449 PMCID: 2363954

55. Gao R, Schellenberg MJ, and Huang SY, et al (2014) Proteolytic degradation of topoisomerase II (Top2) enables the processing of Top2-DNA and Top2-RNA covalent complexes by tyrosyl-DNA-phosphodiesterase 2 (TDP2) J Biol Chem 289 17960-17969 https://doi.org/10.1074/jbc.M114.565374 PMID: 24808172 PMCID: 4140274 
56. Desai SD, Liu LF, and Vazquez-Abad D, et al (1997) Ubiquitin-dependent destruction of topoisomerase I is stimulated by the antitumor drug camptothecin J Biol Chem 272 24159-24164 https://doi.org/10.1074/jbc.272.39.24159 PMID: $\underline{9305865}$

57. Kersting G, Tzvetkov MV, and Huse K, et al (2006) Topoisomerase II beta expression level correlates with doxorubicin-induced apoptosis in peripheral blood cells Naunyn Schmiedebergs Arch Pharmacol 374 21-30 https://doi.org/10.1007/s00210-006-0091-0 PMID: 16957942

58. Ciobotaro P, Drucker L, and Neumann A, et al (2003) The effects of doxorubicin on apoptosis and adhesion molecules of normal peripheral blood leukocytes-an ex vivo study Anticancer Drugs 14 383-389 https://doi.org/10.1097/00001813-200306000-00010 PMID: 12782946

59. Ferraro C, Quemeneur L, and Prigent AF, et al (2000) Anthracyclines trigger apoptosis of both G0-G1 and cycling peripheral blood lymphocytes and induce massive deletion of mature T and B cells Cancer Res 60 1901-1907 PMID: 10766178

60. Lipshultz SE, Anderson LM, and Miller TL, et al (2016) Impaired mitochondrial function is abrogated by dexrazoxane in doxorubicin-treated childhood acute lymphoblastic leukemia survivors: Dana-Farber Cancer Institute Acute Lymphoblastic Leukemia Consortium Cancer 122 946-953 https://doi.org/10.1002/cncr.29872 PMID: 26762648 PMCID: $\underline{477628}$

61. Hasinoff BB, Patel D, and Wu X (2017) Molecular mechanisms of the cardiotoxicity of the proteasomal-targeted drugs bortezomib and carfilzomib Cardiovasc Toxicol 17 237-250 https://doi.org/10.1007/s12012-016-9378-7

62. Hasinoff BB, Wu X, and Patel D, et al (2016) Mechanisms of action and reduced cardiotoxicity of pixantrone; a topoisomerase II targeting agent with cellular selectivity for the topoisomerase lla isoform J Pharmacol Exp Ther 356 397-409 https://doi.org/10.1124/ ipet.115.228650 PMCID: $\underline{4746493}$

63. Hasinoff BB, Patel D, and Wu X (2013) The dual-targeted HER1/HER2 tyrosine kinase inhibitor lapatinib strongly potentiates the cardiac myocyte-damaging effects of doxorubicin Cardiovasc Toxicol 13 33-47 https://doi.org/10.1007/s12012-012-9183-x

64. Vavrova A, Jansova $\mathrm{H}$, and Mackova $\mathrm{E}$, et al (2013) Catalytic inhibitors of topoisomerase II differently modulate the toxicity of anthracyclines in cardiac and cancer cells PLoS One 8 e76676 https://doi.org/10.1371/journal.pone.0076676 PMID: 24116135 PMCID: $\underline{3792022}$

65. Wang X, Ibrahim YF, and Das D, et al (2016) Carfilzomib reverses pulmonary arterial hypertension Cardiovasc Res $110188-199$ https://doi.org/10.1093/cvr/crw047 PMID: 26952044 PMCID: 4836627

66. Al-Harbi NO (2016) Carfilzomib-induced cardiotoxicity mitigated by dexrazoxane through inhibition of hypertrophic gene expression and oxidative stress in rats Toxicol Mech Methods 26 189-195 https://doi.org/10.3109/15376516.2016.1143071 PMID: 26899300

67. Lipshultz SE, Scully RE, and Lipsitz SR, et al (2010) Assessment of dexrazoxane as a cardioprotectant in doxorubicin-treated children with high-risk acute lymphoblastic leukaemia: long-term follow-up of a prospective, randomised, multicentre trial Lancet Oncol 11(10) 950-961 https://doi.org/10.1016/S1470-2045(10)70204-7 PMID: 20850381 PMCID: $\underline{3756093}$

68. McCormack Pharma (2018) Cutting-Edge Medical Education (CEME): A customised association rule learning algorithm for the detection of previously-unknown relationships McCormack Pharma London NW4 4AU UK [www.mccormackpharma.com]

69. Luo X and Kraus WL (2012) On PAR with PARP: cellular stress signaling through poly(ADP-ribose) and PARP-1 Genes Dev 26 417-432 https://doi.org/10.1101/gad.183509.111 PMID: 22391446 PMCID: 3305980

70. Sousa FG, Matuo R, and Soares DG, et al (2012) PARPs and the DNA damage response Carcinogenesis 33 1433-1440 https://doi. org/10.1093/carcin/bgs132 PMID: $\underline{22431722}$

71. Zhang F, Lau SS, and Monks TJ (2012) A dual role for poly(ADP-ribose) polymerase-1 during caspase-dependent apoptosis Toxicol Sci 128(1) 103-114 https://doi.org/10.1093/toxsci/kfs142 PMID: 22523229 PMCID: $\underline{3491962}$ 
72. Kim MY, Zhang T, and Kraus WL (2005) Poly(ADP-ribosyl)ation by PARP-1: 'PAR-laying' NAD+ into a nuclear signal Genes Dev 19 1951-1967 https://doi.org/10.1101/gad.1331805 PMID: 16140981

73. Di Meglio S, Tramontano F, and Cimmino G, et al (2004) Dual role for poly(ADP-ribose)polymerase-1 and -2 and poly(ADPribose)glycohydrolase as DNA-repair and pro-apoptotic factors in rat germinal cells exposed to nitric oxide donors Biochim Biophys Acta 1692 35-44 https://doi.org/10.1016/j.bbamcr.2004.04.002 PMID: 15158362

74. Pacher P, Liaudet L, and Mabley JG, et al (2006) Beneficial effects of a novel ultrapotent poly(ADP-ribose) polymerase inhibitor in murine models of heart failure Int J Mol Med 17 369-375 PMID: 16391839 PMCID: 2245862

75. Szenczi O, Kemecsei P, and Holthuijsen MF, et al (2005) Poly(ADP-ribose) polymerase regulates myocardial calcium handling in doxorubicin-induced heart failure Biochem Pharmacol 69 725-732 https://doi.org/10.1016/j.bcp.2004.11.023 PMID: 15710350 PMCID: 2756478

76. Pacher P, Liaudet L, and Bai P, et al (2002) Activation of poly(ADP-ribose) polymerase contributes to development of doxorubicin-induced heart failure J Pharmacol Exp Ther 300 862-867 https://doi.org/10.1124/jpet.300.3.862 PMID: 11861791

77. Ali M, Kamjoo M, and Thomas HD, et al (2011) The clinically active PARP inhibitor AG014699 ameliorates cardiotoxicity but does not enhance the efficacy of doxorubicin, despite improving tumor perfusion and radiation response in mice Mol Cancer Ther 10 2320-2329 https://doi.org/10.1158/1535-7163.MCT-11-0356 PMID: 21926192 PMCID: 3242069

78. Halder AK, Saha A, and Saha KD, et al (2015) Stepwise development of structure-activity relationship of diverse PARP-1 inhibitors through comparative and validated in silico modeling techniques and molecular dynamics simulation $\mathrm{J}$ Biomol Struct Dyn 33 1756-1779 https://doi.org/10.1080/07391102.2014.969772

79. Saqib U and Baig MS (2014) Probing PARP1-inhibitor complexes for the development of novel inhibitors Cell Mol Bio/ $6043-52$ PMID: 25346248

80. Singh SS, Sarma JA, and Narasu L, et al (2014) A review on PARP1 inhibitors: pharmacophore modeling, virtual and biological screening studies to identify novel PARP1 inhibitors Curr Top Med Chem 14 2020-2030 https://doi.org/10.2174/1568026614666 140929152123 PMID: 25262797

81. Hannigan K, Kulkarni SS, and Bdzhola VG, et al (2013) Identification of novel PARP-1 inhibitors by structure-based virtual screening Bioorg Med Chem Lett 23 5790-5794 https://doi.org/10.1016/j.bmcl.2013.09.007 PMID: 24074844

82. Fatima S, Jatavath MB, and Bathini R, et al (2014) Multiple receptor conformation docking, dock pose clustering and 3D QSAR studies on human poly(ADP-ribose) polymerase-1 (PARP-1) inhibitors J Recept Signal Transduct Res 34 417-430 https://doi.org 110.3109/10799893.2014.917323 PMID: 25046176

83. Steffen JD, Brody JR, and Armen RS, et al (2013) Structural Implications for Selective Targeting of PARPs Front Oncol 3301 https://doi.org/10.3389/fonc.2013.00301

84. Zeng $\mathrm{H}$, Zhang $\mathrm{H}$, and Jang $\mathrm{F}$, et al (2011) Molecular modeling studies on benzimidazole carboxamide derivatives as PARP-1 inhibitors using 3D-QSAR and docking Chem Biol Drug Des 78 333-352 https://doi.org/10.1111/j.1747-0285.2011.01139.x PMID: 21585709

85. Zhang WT, Yan H, and Jiang FC (2007) Construction of pharmacophore model of PARP-1 inhibitor Yao Xue Xue bao 42 279-285 PMID: 17520827

86. Chadha $\mathrm{N}$ and Silakari $\mathrm{O}$ (2017) Pharmacophore based design of some multi-targeted compounds targeted against pathways of diabetic complications J Mol Graph Model 76 412-418 https://doi.org/10.1016/i.jmgm.2017.07.020 PMID: 28802212

87. Baptista SJ, Silva MM, and Moroni E, et al (2017) Novel PARP-1 inhibitor scaffolds disclosed by a dynamic structure-based pharmacophore approach PLoS One 12 e0170846 https://doi.org/10.1371/journal.pone.0170846 PMID: 28122037 PMCID: $\underline{5266331}$ 
88. Chadha $\mathrm{N}$ and Silakari $\mathrm{O}$ (2016) Active site fingerprinting and pharmacophore screening strategies for the identification of dual inhibitors of protein kinase C [Formula: see text] and poly (ADP-ribose) polymerase-1 (PARP-1) Mol Divers 20 747-761 https://doi.org/10.1007/s11030-016-9676-9 PMID: 27216445

89. Schmuck $C$ and Wienand W (2001) Self-complementary quadruple hydrogen-bonding motifs as a functional principle: from dimeric supramolecules to supramolecular polymers Angnew Chem Int Ed Engl 40 4363-4369

90. Prins LJ, Reinhoudt DN, and Timmerman P (2001) Noncovalent synthesis using hydrogen bonding Angew Chem Int Ed Engl 40 2382-2426 PMID: 11443654

91. Mak CH (2016) Unraveling base stacking driving forces in DNA J Phys Chem B 120 6010-6020 https://doi.org/10.1021/acs. jpcb.6b01934 PMID: 27045853

92. Podestà A, Indrieri M, and Brogioli D, et al (2005) Positively charged surfaces increase the flexibility of DNA Biophys J 89 25582563 https://doi.org/10.1529/biophysj.105.064667 PMID: 16040760 PMCID: 1366755

93. Minaga T and Kun E (1983) Probable helical conformation of poly(ADP-ribose). The effect of cations on spectral properties J Biol Chem 258 5726-5730 PMID: $\underline{6853542}$

94. Lait LA, Rutledge LR, and Millen AL, et al (2008) yDNA versus xDNA pyrimidine nucleobases: computational evidence for dependence of duplex stability on spacer location J Phys Chem B 112 12526-12536 https://doi.org/10.1021/jp805547p PMID: 18771305

95. Danilov VI, Dailidonis VV, and van Mourik T, et al (2011) A study of nucleic acid base-stacking by the Monte Carlo method: extended cluster approach Cent Eur J Chem 9 720-727

96. McConnell TL and Wetmore SD (2007) How do size-expanded DNA nucleobases enhance duplex stability? Computational analysis of the hydrogen-bonding and stacking ability of xDNA bases J Phys Chem B 111 2999-3009 https://doi.org/10.1021/ ip0670079 PMID: 17388411

97. Yakovchuk P, Protozanova E, and Frank-Kamenetskii MD (2006) MDBase-stacking and base-pairing contributions into thermal stability of the DNA double helix Nucleic Acids Res 34 564-574 https://doi.org/10.1093/nar/gkj454 PMCID: 1360284

98. Vekilov PG, Feeling-Taylor AR, and Yau ST, et al (2002) Solvent entropy contribution to the free energy of protein crystallization Acta Crystallogr D Biol Crystallogr 58 1611-1616 https://doi.org/10.1107/S0907444902014312 PMID: 12351872

99. Luo R, Gilson HS, and Potter MJ, et al (2001) The physical basis of nucleic acid base stacking in water Biophys J 80 140-148 https://doi.org/10.1016/S0006-3495(01)76001-8 PMID: 11159389 PMCID: 1301220

100. Langlet J, Geissner-Prettre C, and Pullman B, et al (1980) Purine-water interactions in base stacking Quantum Chem 18 $421-437$ https://doi.org/10.1002/qua.560180212

101. Martel $P$ (1979) Base crystallization and base stacking in water Eur J Biochem 96 213-219 https://doi.org/10.1111/j.1432-1033.1979. tb13031.x PMID: 456369

102. Avakyan N, Greschner AA, and Aldaye F, et al (2016) Reprogramming the assembly of unmodified DNA with a small molecule Nat Chem 8 368-376 https://doi.org/10.1038/nchem.2451 PMID: 27001733

103. Tateishi-Karimata $\mathrm{H}$ and Sugimoto $\mathrm{N}$ (2016) Expansion of the DNA alphabet beyond natural DNA recognition Chembiochem 17 1301-1303 https://doi.org/10.1002/cbic.201600181 PMID: 27061868

104.Cafferty BJ, Gállego I, and Chen MC, et al (2013) Efficient self-assembly in water of long noncovalent polymers by nucleobase analogues J Am Chem Soc 135 2447-2450 https://doi.org/10.1021/ja312155v PMID: 23394182 
105.Zhou Z and Bong D (2013) Small-molecule/polymer recognition triggers aqueous-phase assembly and encapsulation Langmuir 29 144-150 https://doi.org/10.1021/la304457y

106. Nowick JS, Cao T, and Noronha G (1994) Molecular recognition between uncharged molecules in aqueous micelles J Am Chem Soc 116 3285-3289 https://doi.org/10.1021/ja00087a014 PMID: 25084234

107.Famulok M, Nowick JS, and Rebek J Jr (1992) Self-replicating systems Acta Chem Scand 46 315-24 https://doi.org/10.3891/acta. chem.scand.46-0315 PMID: 1381212

108. Berger O and Gazit E (2017) Molecular self-assembly using peptide nucleic acids Biopolymers 108 e22930 https://doi.org/10.1002/ bip. 22930

109. McCormack K and George PL (2016) PAR inhibition International Application published under the patent cooperation treaty (PCT) International Application Number PCT/GB2015/054026, International Publication Number WO 2016/051213 A1

110. Allinger NL (2011) Understanding molecular structure from molecular mechanics Comput Aided Mol Des 25 295-316 https://doi. org/10.1007/s10822-011-9422-4

111. Oleinick NL and Evans HH (1985) Poly(ADP-ribose) and the response of cells to lonizing radiation Radiat Res $10129-46$ https:// doi.org/10.2307/3576301 PMID: $\underline{3918331}$

112. Editorial (2005) PLoS Med 2 e272

113. Ioannidis JP (2005) Why most published research findings are false $P$ LoS Med 2 e124 https://doi.org/10.1371/journal.pmed.0020124 PMID: 16060722 PMCID: 1182327

114. Lorenz K (2002) On Aggression (New York: Psychology Press and Taylor and Francis) p 279

115. Rodriguez R, Santiago J, and Fuentes-Vargas, et al (2003) Outstanding neuroprotective efficacy of dexrazoxane in mice subjected to sequential common carotid artery sectioning Drug Dev Res 60 294-302 https://doi.org/10.1002/ddr.10330

116. Hashimoto S, Jing Y, and Kawazoe N, et al (1997) Bufalin reduces the level of topoisomerase II in human leukemia cells and affects the cytotoxicity of anticancer drugs Leuk Res 21 875-883 https://doi.org/10.1016/S0145-2126(97)00061-1 PMID: 9393603

117. Lipshultz SE, Scully RE, and Lipsitz SR, et al (2010) Assessment of dexrazoxane as a cardioprotectant in doxorubicin-treated children with high-risk acute lymphoblastic leukaemia: long-term follow-up of a prospective, randomised, multicentre trial Lancet Oncol 11 950-961 https://doi.org/10.1016/S1470-2045(10)70204-7 PMID: 20850381 PMCID: $\underline{3756093}$

118. Asselin BL, Devidas M, and Chen L, et al (2016) Cardioprotection and safety of dexrazoxane in patients treated for newly diagnosed T-cell acute lymphoblastic leukemia or advanced-stage lymphoblastic non-Hodgkin lymphoma: A Report of the Children's Oncology Group Randomized Trial Pediatric Oncology Group 9404 J Clin Oncol 34 854-862 https://doi.org/10.1200/ JCO.2015.60.8851

119. Reichardt P, Tabone MD, and Mora J, et al (2018) Risk-benefit of dexrazoxane for preventing anthracycline-related cardiotoxicity: re-evaluating the European labelling Future Oncol 14(25): 2663-2676 https://doi.org/10.2217/fon-2018-0210 PMID: 29747541

120. Lebrecht D, Setzer B, and Ketelsen U, etal (2003) Time-dependent and tissue-specific accumulation of mtDNA and respiratory chain defects in chronic doxorubicin cardiomyopathy Circulation 108 2423-2429 https://doi.org/10.1161/01.CIR.0000093196.59829.DF PMID: 14568902

121. Serrano J, Palmeira CM, and Kuehl DW, et al (1999) Cardioselective and cumulative oxidation of mitochondrial DNA following subchronic doxorubicin administration Biochim Biophys Acta 1411 201-205 https://doi.org/10.1016/S0005-2728(99)00011-0 PMID: $\underline{10216166}$

122. Kropp J, Roti Roti EC, and Ringelstetter A, et al (2015) Dexrazoxane diminishes doxorubicin-induced acute ovarian damage and preserves ovarian function and fecundity in mice PLOS One 10 e0142588 https://doi.org/10.1371/journal.pone.0142588 PMID: 26544188 PMCID: 4636352 
123. Szczesny B, Brunyanszki A, and Olah G, et al (2014) Opposing roles of mitochondrial and nuclear DNA integrity: implications for the regulation of mitochondrial function Nucleic Acids Res 42 13161-13173 https://doi.org/10.1093/nar/gku1089 PMID: 25378300 PMCID: $\underline{4245951}$

124. Gupte R, Liu Z, and Kraus WL (2017) PARPs and ADP-ribosylation: recent advances linking molecular functions to biological actions Genes Dev 31 101-126 https://doi.org/10.1101/gad.291518.116 PMID: 28202539 PMCID: 5322727

125. Moreira AC, Branco AF, and Sampaio SF, et al (2014) Mitochondrial apoptosis-inducing factor is involved in doxorubicininduced toxicity on H9c2 cardiomyoblasts Biochim Biophys Acta 1842 2468-2478 https://doi.org/10.1016/j.bbadis.2014.09.015 PMID: 25283819

126. Shin HJ, Kwon HK, and Lee JH, et al (2015) Doxorubicin-induced necrosis is mediated by poly-(ADP-ribose) polymerase 1 (PARP1) but is independent of p53 Sci Rep 515798 https://doi.org/10.1038/srep15798 PMID: 26522181 PMCID: 4629133

127. McCormack K and Brune K (1987) Classical absorption theory and the development of gastric mucosal damage associated with the nonsteroidal antiinflammatory drugs Arch Toxicol 60 261-269 https://doi.org/10.1007/BF01234664 PMID: $\underline{3307703}$

128. McCormack K (1989) Mathematical Model for Assessing Risk of Gastrointestinal Reactions to NSAIDs in Azapropazone, 20 Years of Clinical Use ed KD Rainsford (Lancaster, UK: Kluwer Academic) pp 81-94

129. McCormack K (1994) Non-steroidal anti-inflammatory drugs and spinal nociceptive processing Pain 59 9-43 https://doi. org/10.1016/0304-3959(94)90045-0

130. McCormack K (1994) The spinal actions of NSAIDs and the dissociation between anti-inflammatory and analgesic effects. Proceedings of a meeting at the World Congress on Inflammation '93, Neuronal Plasticity-Implications for pain therapy Drugs 47 28-45 https://doi.org/10.2165/00003495-199400475-00006

131. McCormack K, Prather P, and Chapleo C (1998) Some new insights into the effects opioids in phasic and tonic nociceptive tests Pain 78 79-98 https://doi.org/10.1016/S0304-3959(98)00146-8 PMID: 9839818

132. McCormack K and Chapleo C (1998) Opioid receptors and myocardial protection: do some opioid agonists possess cardioprotective effects? Clin Drug Invest 15 445-454 https://doi.org/10.2165/00044011-199815050-00009

133. McCormack K (1999) Signal transduction in neuropathic pain with special emphasis upon the analgesic role of opioids. Part I. The basic science of phenotype expression in the normal nerve and the regenerating nerve Pain Reviews 6 3-34 https://doi. org/10.1191/096813099676046619

134. McCormack K (1999) Signal transduction in neuropathic pain with special emphasis upon the analgesic role of opioids. Part II. Moving basic science toward a new pharmacotherapy Pain Rev 6 99-131 https://doi.org/10.1191/096813099669853099

135. Gilsbach R, PreissI S, and Grüning BA, et al (2014) Dynamic DNA methylation orchestrates cardiomyocyte development, maturation and disease Nat Commun 55288 https://doi.org/10.1038/ncomms6288 PMID: 25335909 PMCID: 4220495

136. Alpert NR and Mulieri LA (1982) Increased myothermal economy of isometric force generation in compensated cardiac hypertrophy induced by pulmonary artery constriction in the rabbit. A characterization of heat liberation in normal and hypertrophied right ventricular papillary muscles Circ Res $\mathbf{5 0} 491-500$ https://doi.org/10.1161/01.RES.50.4.491 PMID: $\underline{6461437}$

137. Holubarsch C, Goulette RP, and Litten RZ, et al (1985) The economy of isometric force development, myosin isoenzyme pattern and myofibrillar ATPase activity in normal and hypothyroid rat myocardium Circ Res 56 78-86 https://doi.org/10.1161/01. RES.56.1.78 PMID: $\underline{3155672}$

138. Izumo S, Lompré AM, and Matsuoka R, et al (1987) Myosin heavy chain messenger RNA and protein isoform transitions during cardiac hypertrophy. Interaction between hemodynamic and thyroid hormone-induced signals J Clin Invest 79 970-977 https:// doi.org/10.1172/JCI112908 PMID: 2950137 PMCID: 424251 
139. James J, Martin L, and Krenz M, et al (2005) Forced expression of alpha-myosin heavy chain in the rabbit ventricle results in cardioprotection under cardiomyopathic conditions Circulation 111 2339-2346 https://doi.org/10.1161/01.CIR.0000164233.09448. B1 PMID: 15867177 PMCID: 1314981

140. Krenz M and Robbins J (2004) Impact of beta-myosin heavy chain expression on cardiac function during stress J Am Coll Cardiol 44 2390-2397 https://doi.org/10.1016/j.jacc.2004.09.044 PMID: 15607403

141. Blaxall BC, Tschannen-Moran BM, and Milano CA, et al (2003) Differential gene expression and genomic patient stratification following left ventricular assist device support J Am Coll Cardiol 41 1096-1106 https://doi.org/10.1016/S0735-1097(03)00043-3 PMID: 12679207

142. Herron TJ and McDonald KS (2002) Small amounts of alpha-myosin heavy chain isoform expression significantly increase power output of rat cardiac myocyte fragments Circ Res 90 1150-1152 https://doi.org/10.1161/01.RES.0000022879.57270.11 PMID: 12065316

143. Abraham WT, Gilbert EM, and Lowes BD, et al (2002) Coordinate changes in Myosin heavy chain isoform gene expression are selectively associated with alterations in dilated cardiomyopathy phenotype Mol Med 8 750-760 https://doi.org/10.1007/ BF03402039

144. Lowes BD, Gilbert EM, and Abraham WT, et al (2002) Myocardial gene expression in dilated cardiomyopathy treated with betablocking agents N Engl J Med 346 1357-1365 https://doi.org/10.1056/NEJMoa012630 PMID: 11986409

145. Miyata S, Minobe W, and Bristow MR, et al (2000) Myosin heavy chain isoform expression in the failing and nonfailing heart Circ Res 86 386-390 https://doi.org/10.1161/01.RES.86.4.386 PMID: 10700442

146. Lowes BD, Minobe W, and Abraham WT, et al (1997) Changes in gene expression in the intact human heart. Downregulation of alpha-myosin heavy chain in hypertrophied, failing ventricular myocardium J Clin Invest 100 2315-2324 https://doi.org/10.1172/ JCl119770 PMID: 9410910 PMCID: $\underline{508428}$

147. McKinsey TA and Olson EN (2005) Toward transcriptional therapies for the failing heart: chemical screens to modulate genes J Clin Invest 115 538-546 https://doi.org/10.1172/JCI24144 PMID: 15765135 PMCID: 1052006

148. de Beer EL, Bottone AE, and van Der Velden J, et al (2000) Doxorubicin impairs crossbridge turnover kinetics in skinned cardiac trabeculae after acute and chronic treatment Mol Pharmacol 57 1152-1157 PMID: 10825385

149. Bottone, Voest EE, and de Beer EL (1998) Impairment of the actin-myosin interaction in permeabilized cardiac trabeculae after chronic doxorubicin treatment Clin Cancer Res 4 1031-1037 PMID: 9563899

150.de Beer EL, Bottone AE, and van Rijk MC, et al (2002) Dexrazoxane pre-treatment protects skinned rat cardiac trabeculae against delayed doxorubicin-induced impairment of crossbridge kinetics Br J Pharmacol 135 1707-1714 https://doi.org/10.1038/ sj.bjp.0704621 PMID: 11934811 PMCID: 1573287

151.Ferreira A, Cunha-Oliveira T, and Simões RF, et al (2017) Altered mitochondrial epigenetics associated with subchronic doxorubicin cardiotoxicity Toxicology 390 63-73 https://doi.org/10.1016/j.tox.2017.08.011 PMID: 28865727

152. Hang CT, Yang J, and Han P, et al (2010) Chromatin regulation By Brg1 underlies heart muscle development and disease Nature 466 62-67 https://doi.org/10.1038/nature09130 PMID: 20596014 PMCID: 2898892

153. Schreiber V, Dantzer F, and Ame JC, et al (2006) Poly(ADP-ribose): novel functions for an old molecule Nat Rev Mol Cell Biol 7 517-528 https://doi.org/10.1038/nrm1963 PMID: 16829982

154. Bartha E, Solti I, and Kereskai L, et al (2009) PARP inhibition delays transition of hypertensive cardiopathy to heart failure in spontaneously hypertensive rats Cardiovasc Res 83 501-510 https://doi.org/10.1093/cvr/cvp144 PMID: 19443425 
155.Pillai JB, Gupta M, and Rajamohan SB, et al (2006) Poly(ADP-ribose)-1-deficient mice are protected from angiotensin IIinduced cardiac hypertrophy Am J Physiol Heart Circ Physiol 291 H1545-H1553 https://doi.org/10.1152/ajpheart.01124.2005 PMID: 16632544

156.Pillai JB, Russell HM, and Raman J, et al (2005) Increased expression of poly(ADP-ribose) polymerase-1 contributes to caspase-independent myocyte cell death during heart failure Am J Physiol Heart Circ Physiol 288 H486-H496 https://doi.org/10.1152/ ajpheart.00437.2004

157. Swain SM, Whaley FS, and Gerber MC, et al (1997) Cardioprotection with dexrazoxane for doxorubicin-containing therapy in advanced breast cancer J Clin Oncol 15 1318-1332 https://doi.org/10.1200/JCO.1997.15.4.1318 PMID: $\underline{9193323}$

158. Tebbi CK, London WB, and Friedman D, et al (2007) Dexrazoxane-associated risk for acute myeloid leukemia/myelodysplastic syndrome and other secondary malignancies in pediatric Hodgkin's disease J Clin Oncol 25 493-500 https://doi.org/10.1200/ $\underline{\text { JCO.2005.02.3879 PMID: } 17290056}$

159.van Dalen EC, van den Berg H, and Raphaël MF, et al (2011) Should anthracyclines and dexrazoxane be used for children with cancer? Lancet Oncol 12 12-13 https://doi.org/10.1016/S1470-2045(10)70301-6 PMID: 21195321

160.Swain SM, Whaley FS, and Gerber MC, et al (1997) Delayed administration of dexrazoxane provides cardioprotection for patients with advanced breast cancer treated with doxorubicin-containing therapy J Clin Oncol 15 1333-1340 https://doi. org/10.1200/JCO.1997.15.4.1333 PMID: $\underline{9193324}$

161.van Dalen EC, Caron HN, and Dickinson HO, et al (2008) Cardioprotective interventions for cancer patients receiving anthracyclines Cochrane Database Syst Rev (2): CD003917 [doi:10.1002/14651858.CD003917.pub3] PMID: 18425895

162. Jirkovsky E, Lencova O, and Jirkovska A, et al (2014) Study of molecular mechanisms involved in cardioprotective action of dexrazoxane against anthracycline cardiotoxicity in rabbits Heart 100(Suppl 4) A7-A8 https://doi.org/10.1136/heartjnl-2014-306916.22

163. Lencova-Popelova O, Jirkovsky E, and Jansova H, et al (2016) Cardioprotective effects of inorganic nitrate/nitrite in chronic anthracycline cardiotoxicity: comparison with dexrazoxane J Mol Cell Cardiol 91 92-103 https://doi.org/10.1016/i.yimcc.2015.12.021 PMID: 26724189

164. Bures J, Jirkovska A, and Sestak V, et al (2017) Investigation of novel dexrazoxane analogue JR-311 shows significant cardioprotective effects through topoisomerase Ilbeta but not its iron chelating metabolite Toxicology 392 1-10 https://doi.org/10.1016/j. tox.2017.09.012 PMID: 28941780

165. Yeh ETH (2017) Doxorubicin-induced cardiotoxicity: the role of topoisomerase $2 \beta$ National Institute of Health (NIH) Funding Application \#9246567. Project: \#5R01HL126916-04

166. Yeh ETH (2016) Doxorubicin-induced cardiotoxicity: the role of topoisomerase $2 \beta$ National Institute of Health (NIH) Funding Application \#9335618. Project: \#7R01HL126916-03

167. Yeh ETH (2016) Doxorubicin-induced cardiotoxicity: the role of topoisomerase $2 \beta$ National Institute of Health (NIH) Funding Application \#9042425. Project: \#5R01HL126916-02

168. Yeh ETH (2015) Doxorubicin-induced cardiotoxicity: the role of topoisomerase $2 \beta$ National Institute of Health (NIH) Funding Application \#8860807. Project: \#1R01HL126916-01

169. Hasinoff BB, Kuschak TI, and Yalowich JC, et al (1995) A QSAR study comparing the cytotoxicity and DNA topoisomerase II inhibitory effects of bisdioxopiperazine analogs of ICRF-187 (dexrazoxane) Biochem Pharmacol 50 953-958 https://doi. org/10.1016/0006-2952(95)00218-O PMID: $\underline{7575679}$

170. Herman EH, Mhatre RM, and Chadwick DP (1974) Modification of some of the toxic effects of daunorubicin (NSC-82,151) by pretreatment with the antineoplastic agent ICRF 159 (NSC-129,943) Toxicol Appl Pharmacol 27 517-526 https://doi.org/10.1016/0041$\underline{008 \times(74) 90031-3}$ 
171. Herman EH, Ardalan B, and Bier C, et al (1979) Reduction of daunorubicin lethality and myocardial cellular alterations by pretreatment with ICRF-187 in Syrian golden hamsters Cancer Treat Rep 63 89-92 PMID: 421236

172. Herman EH and Ferrans VJ (1983) Influence of vitamin E and ICRF-187 on chronic doxorubicin cardiotoxicity in miniature swine Lab Invest 49 69-77 PMID: $\underline{6408310}$

173. Herman EH, Ferrans VJ, and Young RS, et al (1988) Pretreatment with ICRF-187 allows a marked increase in the total cumulative dose of doxorubicin tolerated by beagle dogs Drugs Exp Clin Res 14 563-570 PMID: 3147886

174. Herman EH and Ferrans VJ (1998) Preclinical animal models of cardiac protection from anthracycline-induced cardiotoxicity Semin Oncol 25(4) 15-21 PMID: $\underline{9768819}$

175. Takemura G and Fujiwara H (2007) Doxorubicin-induced cardiomyopathy from the cardiotoxic mechanisms to management Prog Cardiovasc Dis 49 330-352 https://doi.org/10.1016/j.pcad.2006.10.002 PMID: 17329180

176. Singal PK, Deally CM, and Weinberg LE (1987) Subcellular effects of Adriamycin in the heart: a concise review J Mol Cell Cardiol 19 817-828 https://doi.org/10.1016/S0022-2828(87)80392-9 PMID: 3320376

177. Kalyanaraman B, Perez-Reyes E, and Mason RP (1980) Spin-trapping and direct electron spin resonance investigations of the redox metabolism of quinone anticancer drugs Biochim Biophys Acta 630 119-130 https://doi.org/10.1016/0304-4165(80)90142-7 PMID: $\underline{6248123}$

178. Doroshow JH (1983) Effect of anthracycline antibiotics on oxygen radical formation in rat heart Cancer Res $43460-472$

179. Gutteridge JMC (1984) Lipid peroxidation and possible hydroxyl radical formation stimulated by the self-reduction of a doxorubicin-iron (III) complex Biochem Pharmacol 33 1725-1728 https://doi.org/10.1016/0006-2952(84)90340-X PMID: 6329216

180. Ichikawa Y, Ghanefar M, and Bayeva R, et al (2014) Cardiotoxicity of doxorubicin is mediated through mitochondrial iron accumulation J Clin Invest 124 617-630 https://doi.org/10.1172/JCI72931 PMID: 24382354 PMCID: 3904631

181. Gammella E, Maccarinelli F, and Buratti P, et al (2014) The role of iron in anthracycline cardiotoxicity Drug Metab Transp 525

182. Hoffbrand AV, Cohen A, and Hershko C (2003) Role of deferiprone in chelation therapy for transfusional iron overload Blood 102 17-24 https://doi.org/10.1182/blood-2002-06-1867 PMID: 12637334

183. Glickstein H, El RB, and Link G, et al (2006) Action of chelators in iron-overloaded cells: Accessibility to intracellular labile iron and functional consequences Blood 108 3195-3203 https://doi.org/10.1182/blood-2006-05-020867 PMID: 16835377

184. Simunek T, Klimtova I, and Kaplanova J, et al (2004) Rabbit model for in vivo study of anthracycline-induced heart failure and for the evaluation of protective agents Eur J Heart Fail 6 377-387 https://doi.org/10.1016/j.ejheart.2003.05.003

185. Popelova O, Sterba M, and Simunek T, et al (2008) Deferiprone does not protect against chronic anthracycline cardiotoxicity in vivo J Pharmacol Exp Ther 326 259-269 https://doi.org/10.1124/jpet.108.137604 PMID: 18434588

186. Hasinoff BB, Patel D, and Wu X (2003) The oral iron chelator ICL670A (desferasirox) does not protect myocytes against doxorubicin Free Radic Biol Med 35 1469-1479 https://doi.org/10.1016/j.freeradbiomed.2003.08.005 PMID: 14642395

187. McCormack K and Davies $\mathrm{R}$ (1996) The enigma of potassium ion in the management of dentine hypersensitivity. Is nitric oxide the elusive second messenger? Pain 68 5-11 https://doi.org/10.1016/S0304-3959(96)03142-9 